\title{
Asymptotic structure of the Rarita-Schwinger theory in four spacetime dimensions at spatial infinity
}

\author{
Oscar Fuentealba, ${ }^{a}$ Marc Henneaux, ${ }^{a, b}$ Sucheta Majumdar, ${ }^{a}$ Javier Matulich ${ }^{a}$ \\ and Turmoli Neogi ${ }^{a}$ \\ ${ }^{a}$ Université Libre de Bruxelles and International Solvay Institutes, \\ ULB-Campus Plaine CP231, B-1050 Brussels, Belgium \\ ${ }^{b}$ Collège de France, \\ 11 place Marcelin Berthelot, 75005 Paris, France \\ E-mail: ofuentea@ulb.ac.be, henneaux@ulb.ac.be, \\ sucheta.majumdar@ulb.ac.be, jmatulic@ulb.ac.be, \\ turmoli.neogi@ulb.ac.be
}

ABSTRACT: We investigate the asymptotic structure of the free Rarita-Schwinger theory in four spacetime dimensions at spatial infinity in the Hamiltonian formalism. We impose boundary conditions for the spin-3/2 field that are invariant under an infinite-dimensional (abelian) algebra of non-trivial asymptotic fermionic symmetries. The compatibility of this set of boundary conditions with the invariance of the theory under Lorentz boosts requires the introduction of boundary degrees of freedom in the Hamiltonian action, along the lines of electromagnetism. These boundary degrees of freedom modify the symplectic structure by a surface contribution appearing in addition to the standard bulk piece. The Poincaré transformations have then well-defined (integrable, finite) canonical generators. Moreover, improper fermionic gauge symmetries, which are also well-defined canonical transformations, are further enlarged and turn out to be parametrized by two independent angle-dependent spinor functions at infinity, which lead to an infinite-dimensional fermionic algebra endowed with a central charge. We extend next the analysis to the supersymmetric spin- $(1,3 / 2)$ and spin- $(2,3 / 2)$ multiplets. First, we present the canonical realization of the super-Poincaré algebra on the spin-(1,3/2) multiplet, which is shown to be consistently enhanced by the infinite-dimensional abelian algebra of angle-dependent bosonic and fermionic improper gauge symmetries associated with the electromagnetic and the Rarita-Schwinger fields, respectively. A similar analysis of the spin- $(2,3 / 2)$ multiplet is then carried out to obtain the canonical realization of the super-Poincaré algebra, consistently enhanced by the abelian improper bosonic gauge transformations of the spin-2 field (BMS supertranslations) and the abelian improper fermionic gauge transformations of the spin-3/2 field.

Keywords: Gauge Symmetry, Global Symmetries, Space-Time Symmetries, Classical Theories of Gravity

ArXiv EPrint: 2011.04669 


\section{Contents}

1 Introduction 1

2 Formulation of the Rarita-Schwinger theory with standard asymptotic conditions 4

3 New asymptotic conditions for the Rarita-Schwinger field 6

$\begin{array}{ll}3.1 & \text { Formulation of the new conditions }\end{array}$

3.2 Finiteness of the kinetic term 8

3.3 Equations of motion $\quad 9$

$\begin{array}{lll}3.4 & \text { Pre-symplectic form } & 10\end{array}$

3.5 Canonical realization of the boosts - Need for a new surface field 12

$\begin{array}{ll}3.6 & \text { Poincaré generators } \\ 3.7 & 15\end{array}$

3.7 A twofold of infinite-dimensional fermionic improper gauge symmetries $\quad 16$

$\begin{array}{lll}3.8 & \text { Asymptotic symmetry algebra } & 17\end{array}$

4 Rigid supersymmetry: spin- $\left(1, \frac{3}{2}\right)$ multiplet $\quad 18$

$\begin{array}{lll}4.1 \text { Invariance under rigid supersymmetry } & 19\end{array}$

$\begin{array}{ll}\text { 4.2 Canonical realization of the asymptotic symmetries } & 19\end{array}$

4.2.1 Super-Poincaré algebra 20

4.2.2 Infinite-dimensional algebra of improper gauge symmetries 21

5 Rigid supersymmetry: spin- $\left(2, \frac{3}{2}\right)$ multiplet $\quad 22$

5.1 Invariance under rigid supersymmetry 23

5.2 Canonical realization of the asymptotic symmetries 23

5.2.1 Super-BMS algebra of $[14,15] \quad 23$

$\begin{array}{ll}\text { 5.2.2 Infinite-dimensional fermionic gauge algebra } & 25\end{array}$

6 Conclusions 26

$\begin{array}{lr}\text { A Notations and conventions } & 26\end{array}$

B Asymptotic conditions and charges in spherical coordinates 28

\section{Introduction}

The asymptotic structure of gravity is an extremely rich subject, which has attracted a revived interest in the last years [1].

One of the lessons that has been learned from the study of the behaviour of the gravitational field at infinity is that the algebra of asymptotic symmetries can be much larger than the algebra of background isometries. The first instance where this phenomenon 
was observed was four-dimensional Einstein gravity with vanishing cosmological constant, where studies at null infinity revealed that the asymptotic symmetries formed the infinitedimensional Bondi-van der Burg-Metzner-Sachs (BMS) algebra [2-8], which can even be further extended to include super-rotations [9-12]. Another much studied example is antide Sitter gravity in three spacetime dimensions, where the asymptotic symmetry algebra is given, for standard AdS boundary conditions, by two copies of the infinite-dimensional Virasoro algebra [13]. In both cases, the asymptotic symmetry algebra contains the algebra of background isometries (the Poincaré algebra or the anti-de Sitter algebra, respectively), but is strictly bigger than it. Furthermore, a non trivial central charge with interesting physical significance may appear in the Poisson bracket algebra of the generators of the asymptotic symmetries - which is necessarily trivial for the subalgebra of background isometries - , as it is the case for three-dimensional AdS gravity [13].

When one considers the supersymmetric versions of these two theories, one finds in both cases a graded extension of the respective infinite-dimensional symmetry algebras. This was shown to be the case for supergravity in four dimensions in [14], where a graded extension of the BMS algebra was exhibited to be a symmetry of the theory. While the analysis of [14] was performed at null infinity, it was later confirmed in [15] that the same superalgebra emerges at spatial infinity. In the three-dimensional context, the infinite-dimensional asymptotic symmetry superalgebras of anti-de Sitter supergravities were worked out in [16, 17] and identified with (nonlinear) extended super-conformal algebras.

The main motivation of our paper, which deals with asymptotically flat spaces in four spacetime dimensions, arises from the following puzzle. A notable feature of the supersymmetric extension of the BMS algebra of [14] is that it contains only a finite number of fermionic generators. However, group theoretical arguments indicate that extensions of the BMS algebra by an infinite number of fermionic generators exist [14] and indeed, such super-BMS algebras appeared more recently in the analysis of supergravity at null infinity [18-22]. The question we would like to explore is whether one can develop a consistent Hamiltonian formulation of this infinite-dimensional fermionic extension. Such a step would shed important light on the quantum formulation of the theory.

Indications that it should be possible to implement consistently such an extension comes actually from asymptotically flat supergravity in three dimensions, where a super$\mathrm{BMS}_{3}$ algebra possessing this feature was uncovered [23] (see also [24-30] for extended supergravity).

By consistent Hamiltonian formulation of the symmetry, we specifically mean the following: can one impose boundary conditions on the phase space variables of supergravity so that the super-BMS transformations have a well-defined moment map?

The phase space variables of supergravity are fields defined on a Cauchy hypersurface (or, which is sufficient for our purposes, "asymptotically Cauchy hypersurface"), providing thereby complete initial data out of which the future (Cauchy) development, including the behaviour of the fields near null infinity, can in principle be derived. The boundary conditions are given at large distances on these Cauchy hypersurfaces, i.e., at spatial infinity.

A first requirement to be imposed is that the boundary conditions make the action, and in particular, its kinetic term finite. This guarantees the existence of a well-defined, finite, 
symplectic 2-form leading to the standard Hamiltonian structure. A second requirement is that the symmetries under investigation - here, super-BMS transformations - not only preserve the boundary conditions, but also the symplectic form and hence can be associated with a well-defined canonical generator.

This program was successfully achieved for the BMS group of general relativity in [3133], where boundary conditions implementing the above requirements were given. In fact, two inequivalent sets of boundary conditions consistent with the BMS symmetry were devised. The development of initial data satisfying either of the boundary conditions at spatial infinity was investigated near null infinity, confirming incidentally as a by-product that the existence of a smooth null infinity resulting from regular initial data on a Cauchy hypersurface is far from granted - as forcefully stressed and vigorously studied in [34, 35] and references therein. A similar analysis was performed in electromagnetism for the angledependent $u(1)$ transformations [36].

The question, then, is whether one can go beyond the supergravity analysis of [15] by relaxing the boundary conditions given there in such a way that an infinite-dimensional fermionic extension (and not just a finite-dimensional one) of the BMS symmetry can be realized as asymptotic symmetry, with well-defined canonical generators.

The problem is rather intricate because it involves on the bosonic side a generalized form of parity conditions [31-33,37]. We shall therefore first consider the simpler linearized models, which contain in the gravitational case already a good wealth of information on the interacting case [38] (but see [39] for the Yang-Mills theory). Free supersymmetric models are the scope of this paper. In a subsequent work we shall come back to the full interacting theories.

We first show that the free Rarita-Schwinger field on a Minkowski background admits, with suitable boundary conditions that are verified to be Poincaré invariant, an infinite number of non-trivial fermionic improper [40] gauge symmetries parametrized by two independent functions of the angles. These form an abelian algebra with a non trivial central charge. The construction needs the introduction of surface degrees of freedom, which we describe. These are analogous to the surface degrees of freedom needed for a consistent Lorentz-invariant description of electromagnetism [36] (see also [41]) and are somewhat reminiscent of the edge modes of [42-44].

We then consider the free supersymmetric $\left(1, \frac{3}{2}\right)$ and $\left(2, \frac{3}{2}\right)$ multiplets and show that rigid supersymmetry is compatible with our boundary conditions, leading to an infinite set of fermionic symmetries (standard rigid supersymmetry and improper fermionic gauge symmetries). The algebra of the charges is computed and the Lorentz transformation properties of the fermionic parameters are in particular written.

Our paper is organized as follows. In section 2, we recall the results of [15] in the context of the free Rarita-Schwinger field and stress that with the boundary conditions adopted in this paper, there is no non trivial fermionic asymptotic symmetry. We next analyze (section 3) the free Rarita-Schwinger theory with softened boundary conditions involving an improper gauge term. We show that these conditions are compatible with Poincaré invariance - specifically, the boosts — if one add a surface field with specific Lorentz transformations. This surface field enters the symplectic form through a surface 
integral at infinity, much in the same way as what was shown for electromagnetism in [36]. We then work out the Poincaré generators, which are all well-defined (integrable and finite), as well as the generators of the fermionic improper gauge symmetries, which turn out to form an abelian superalgebra parametrized by two spinor functions of the angles, with a non trivial central extension. In section $\mathbf{4}$, we extend the analysis to the spin- $(1,3 / 2)$ multiplet and show compatibility with supersymmetry. This requires to define the supersymmetry transformations by including in them a field-dependent $u(1)$ gauge transformation, in order to leave the (pre-)symplectic form invariant. In section $\mathbf{5}$, we achieve the same task for the spin- $(2,3 / 2)$ multiplet. We show that the algebra of the rigid symmetries and the improper gauge symmetries form a graded extension of the BMS algebra possessing an infinite number of fermionic generators - the subalgebra of rigid symmetries and bosonic improper gauge symmetries being isomorphic to the super-BMS algebra of $[14,15]$. Finally, section 6 gives some concluding remarks. Two appendices provide conventions and some more technical material.

\section{Formulation of the Rarita-Schwinger theory with standard asymptotic conditions}

We consider the Rarita-Schwinger field $\psi_{\mu}$ in four-dimensional Minkowski space. In the canonical formalism, the dynamical variables are its spatial components $\psi_{k}$ and the action is given in flat coordinates by [45]

$$
S=-\frac{i}{2} \int d^{4} x \bar{\psi}_{\mu} \gamma^{\mu \nu \rho} \partial_{\nu} \psi_{\rho}+\text { "boundary terms" }=\int d t\left(K-H-i \int d^{3} x \psi_{0}^{T} \mathcal{S}\right) .
$$

The kinetic term in the action reads explicitly ${ }^{1}$

$$
K=\frac{i}{2} \int d^{3} x \psi_{k}^{T} \gamma^{k m} \partial_{t} \psi_{m}+B
$$

where $B$ is a surface term chosen so that the action is finite. Its explicit form depends on the boundary conditions on the spin- $\frac{3}{2}$ field. The Hamiltonian is given by

$$
H=\frac{i}{2} \int d^{3} x \psi_{k}^{T} \gamma_{0} \gamma^{k m n} \partial_{m} \psi_{n}
$$

The constraint $\mathcal{S}$ enforced by varying the Lagrange multiplier $\psi_{0}$ is

$$
\mathcal{S}=\gamma^{m n} \partial_{m} \psi_{n} \approx 0
$$

The standard asymptotic conditions on $\psi_{k}$ are

$$
\psi_{k}=\mathcal{O}\left(\frac{1}{r^{2}}\right)
$$

\footnotetext{
${ }^{1}$ We choose the convention where Dirac conjugate for Majorana spinors reads $\bar{\psi}_{\mu}=\psi_{\mu}^{T} \gamma_{0}$ with all $\gamma$-matrices real, $\gamma_{0}$ being antisymmetric and $\gamma_{i}$ symmetric.
} 
With these boundary conditions, the boundary term $B$ can be taken to vanish and the kinetic term in the action is finite. It implies the bracket relation

$$
\left\{\psi_{m}^{\alpha}(x), \psi_{n}^{\beta}(\bar{x})\right\}=\frac{i}{2}\left(\gamma_{n} \gamma_{m}\right)^{\alpha \beta} \delta^{(3)}(x-\bar{x})
$$

showing that $\psi_{k}$ is somehow canonically self-conjugate.

The Lagrange multiplier $\psi_{0}$ describes the gauge transformation involved in the time evolution of the system and is arbitrary. We shall impose

$$
\psi_{0}=\mathcal{O}\left(\frac{1}{r^{2}}\right)
$$

so that the motion "involves no gauge transformation at infinity". This is a convenient asymptotic gauge fixing condition, which clearly makes the constraint term in the action finite.

The Hamiltonian action (2.1) is invariant under infinitesimal Poincaré transformations obtained by taking the Poisson bracket of the fields with the Poincaré generators

$$
\int d^{3} x\left(\xi \mathcal{E}+\xi^{m} \mathcal{P}_{m}\right)
$$

where

$$
\begin{aligned}
\mathcal{E} & =\frac{i}{2}\left[\psi_{k}^{T} \gamma^{k m n} \gamma_{0} \partial_{m} \psi_{n}+\partial_{k}\left(\psi_{j}^{T} \gamma^{j} \gamma_{0} \psi^{k}\right)\right], \\
\mathcal{P}_{k} & =-i\left[\frac{1}{2} \partial_{m}\left(\psi_{n}^{T} \gamma^{m n} \psi_{k}\right)+\frac{1}{2} \psi_{m}^{T} \gamma^{m n} \partial_{k} \psi_{n}+\frac{1}{8} \partial_{p}\left(\psi_{m}^{T} \gamma^{m n} \gamma_{k}{ }^{p} \psi_{n}\right)\right] .
\end{aligned}
$$

Here,

$$
\begin{aligned}
\xi & =b_{i} x^{i}+a^{\perp}, \\
\xi^{i} & =b^{i}{ }_{j} x^{j}+a^{i},
\end{aligned}
$$

are the components of the vector fields that parametrize the Poincare transformations. The arbitrary constants $b^{i}$ and $b_{i j}=-b_{j i}$ parametrize the boosts and spatial rotations, respectively. The constants $a^{\perp}$ and $a^{i}$ stand for standard translations (the term $b^{i} x^{0}$ in $\xi^{i}$ can be absorbed in $a^{i}$ at any fixed time). Note that the integral giving the Poincaré generators converges at infinity even for boosts and spatial rotations since in that case the integrand behaves as $\sim \frac{1}{r^{4}}$.

One gets

$$
\begin{aligned}
\delta_{\xi} \psi_{p} & =\left\{\psi_{p}, \int d^{3} x\left(\xi \mathcal{E}+\xi^{m} \mathcal{P}_{m}\right)\right\} \\
& =\xi \gamma_{p}{ }^{j k} \gamma_{0} \partial_{j} \psi_{k}+\frac{1}{2} \partial_{j} \xi \gamma^{j} \gamma_{0} \psi_{p}-\frac{1}{2} \xi \gamma_{p} \gamma_{0} \mathcal{S}+\mathcal{L}_{\xi} \psi_{p}
\end{aligned}
$$

where the Lie derivative of $\psi_{k}$ is given by

$$
\mathcal{L}_{\xi} \psi_{k}=\xi^{m} \partial_{m} \psi_{k}+\partial_{k} \xi^{m} \psi_{m}+\frac{1}{4} \partial_{m} \xi_{n} \gamma^{m n} \psi_{k}
$$


By construction, this transformation leaves the symplectic form invariant since it is a canonical transformation. Furthermore, it is direct to check that it leaves the constraint invariant, $\delta_{\xi} \mathcal{S} \approx 0$.

The Hamiltonian action (2.1) is also invariant under fermionic gauge transformations of the form

$$
\delta_{\eta} \psi_{k}=\partial_{k} \eta
$$

where $\partial_{k} \eta$ must be of order $r^{-2}$ in order to preserve the asymptotic decay of the spin- $\frac{3}{2}$ field, i.e.,

$$
\eta=\eta_{0}+\mathcal{O}\left(\frac{1}{r}\right)
$$

the constant term $\eta_{0}$ being ineffective in (2.15). This transformation is again canonical, being generated by

$$
Q[\eta]=i \int d^{3} x\left(\eta-\eta_{0}\right)^{T} \mathcal{S}
$$

an expression that can easily be verified again to converge at infinity. One has indeed

$$
\delta_{\eta} \psi_{k}=\left\{\psi_{k}, Q[\eta]\right\} .
$$

The generator $Q[\eta]$ can be rewritten as

$$
Q[\eta]=i \int d^{3} x \eta^{T} \mathcal{S}-i \eta_{0}^{T} \oint d^{2} S_{k} \gamma^{k m} \psi_{m},
$$

since $-\int d^{3} x \mathcal{S}+\oint d^{2} S_{k} \gamma^{k m} \psi_{m}=0$ (Stokes theorem). When the constraints hold, the generator $Q[\eta]$ vanishes and so, the gauge transformations are all proper [40]. This is also true for the zero mode $\eta_{0}$, as it should, since $\eta_{0}$ drops from the gauge transformations. In the presence of couplings, of course, the constraint-generator $\mathcal{S}$ is not linear anymore. There is a source contribution that makes the surface integral multiplying $\eta_{0}$ non-zero. But even in that case, there are only four improper fermionic gauge transformations parametrized by the zero mode $\eta_{0}$ because the generators of the other fermionic gauge symmetries are (weakly) equal to zero [15].

The absence of improper gauge transformations in the free theory is due to our choice of $\mathcal{O}\left(r^{-2}\right)$ fall-off of the Rarita-Schwinger field. We shall now discuss less restrictive conditions that lead to a much more interesting asymptotic symmetry structure.

\section{New asymptotic conditions for the Rarita-Schwinger field}

\subsection{Formulation of the new conditions}

The less restrictive boundary conditions developed in this paper allow for a $\mathcal{O}\left(r^{-1}\right)$ term in the gravitino field $\psi_{k}$. However, if one were to consider an arbitrary $\mathcal{O}\left(r^{-1}\right)$ behaviour, one would encounter divergences in the symplectic structure and in the Lorentz generators that are difficult to tame. For that reason, the allowed $\mathcal{O}\left(r^{-1}\right)$ in $\psi_{k}$ will be assumed to take the specific form of a gradient. We therefore adopt as asymptotic conditions

$$
\psi_{k}=\partial_{k} \chi+\mu_{k}
$$


where

$$
\chi=\bar{\chi}+\mathcal{O}\left(\frac{1}{r}\right)
$$

and

$$
\mu_{k}=\frac{\bar{\mu}_{k}}{r^{2}}+\mathcal{O}\left(\frac{1}{r^{3}}\right)
$$

where the coefficients of the various powers of $r$ are functions of the angles. We call $\psi_{k}$ the "complete gravitino field" and refer to $\mu_{k}$ as the "bulk field" and $\bar{\chi}$ as the "surface field". The bulk field $\mu_{k}$ depends on all three spatial coordinates $x^{k}$, while $\bar{\chi}$ depends only on the angles of the 2-sphere "at infinity". It is convenient to extend, as we have done, the surface field into a bulk field $\chi$. There is clearly some ambiguity in the process since higher powers in $r^{-1}$ in $\chi$ can be absorbed into $\mu_{k}$, but this will not be a problem (this redundancy will appear as a proper gauge symmetry).

The inclusion of the surface field $\bar{\chi}$ in the boundary condition is the new feature with respect to the previous treatment of [15]. One might argue at this point that because the new term $\partial_{k} \chi$ in the asymptotic behaviour of $\psi_{k}$ is a gradient, it should be irrelevant since it takes the form of a gauge transformation. However, only proper gauge transformations correspond to redundancies in the description of the system [40]. Improper gauge transformations, which have non-vanishing generators even on-shell, do change the physical state of the system. The added gradient term $\partial_{k} \bar{\chi}$ does turn out to be an improper gauge transformation. A similar extension of the boundary conditions by an improper gauge term that is the leading term in the asymptotic expansion of the field was considered earlier in [46].

The boundary conditions on $\mu_{k}$ will actually need to be strengthened in order for the kinetic term in the action to be finite. Anticipating what we shall find below, we already impose the needed extra condition, which expresses that the constraint function $\mathcal{S}$ should decay one power of $r^{-1}$ faster than what generically follows from the boundary conditions on the fields, i.e., it should decay as $r^{-4}$. Thus we impose

$$
\mathcal{S}=\mathcal{O}\left(\frac{1}{r^{4}}\right)
$$

which is very reminiscent of the fall-off conditions on the constraints in the case of electromagnetism [36] and gravity [31, 33].

The new asymptotic conditions on the gravitino field are easily checked to be preserved under Poincaré transformations, which we take to coincide with (2.13),

$$
\delta_{\xi} \psi_{p}=\xi \gamma_{p}^{j k} \gamma_{0} \partial_{j} \psi_{k}+\frac{1}{2} \partial_{j} \xi \gamma^{j} \gamma_{0} \psi_{p}-\frac{1}{2} \xi \gamma_{p} \gamma_{0} \mathcal{S}+\mathcal{L}_{\xi} \psi_{p}
$$

leading to

$$
\begin{aligned}
\delta_{\xi} \chi & =-\xi \gamma_{0} \gamma^{m} \partial_{m} \chi+\frac{1}{2} \partial_{j} \xi \gamma^{j} \gamma_{0} \chi+\mathcal{L}_{\xi} \chi, \\
\delta_{\xi} \mu_{p} & =\xi \gamma_{p}{ }^{j k} \gamma_{0} \partial_{j} \mu_{k}+\frac{1}{2} \partial_{j} \xi \gamma^{j} \gamma_{0} \mu_{p}-\frac{1}{2} \xi \gamma_{p} \gamma_{0} \gamma^{j k} \partial_{j} \mu_{k}+\mathcal{L}_{\xi} \mu_{p} .
\end{aligned}
$$

Since the split of $\psi_{p}$ into $\partial_{p} \chi$ and $\mu_{p}$ involves ambiguities, there is also some ambiguity in the expressions for $\delta_{\xi} \chi$ and $\delta_{\xi} \mu_{p}$, besides the usual gauge freedom. The above choice, such that $\delta_{\xi} \chi$ and $\delta_{\xi} \mu_{p}$ depend respectively only on $\chi$ and $\mu_{p}$, is particularly convenient. 
The boundary conditions are also preserved by gauge transformations of the form

$$
\delta_{\epsilon} \chi=\epsilon, \quad \delta_{\varepsilon} \mu_{m}=\partial_{m} \varepsilon,
$$

spanned by two fermionic parameters, $\epsilon$ and $\varepsilon$, whose asymptotic behaviors are given by

$$
\begin{aligned}
\epsilon & =\bar{\epsilon}+\mathcal{O}\left(\frac{1}{r}\right), \\
\varepsilon & =\frac{\bar{\varepsilon}}{r}+\mathcal{O}\left(\frac{1}{r^{2}}\right) .
\end{aligned}
$$

As we shall see, these transformations involve both proper and improper gauge symmetries.

\subsection{Finiteness of the kinetic term}

That the addition of a gradient of order $\mathcal{O}\left(r^{-1}\right)$ to the gravitino field is a non-trivial step can be immediately seen at two places. First, the action - specifically the kinetic term - is superficially divergent. Second, the integral giving the Lorentz generators also superficially diverges at infinity (logarithmically) since the integrand, which is not gauge invariant, behaves now as $r^{-3}$. We examine these two problems successively, starting with a proper definition of the action.

If one decomposes the Hamiltonian kinetic term (2.2), one gets three kind of terms:

$$
\frac{i}{2} \int d^{3} x \partial_{k} \chi^{T} \gamma^{k m} \partial_{m} \dot{\chi}
$$

which is formally linearly divergent;

$$
\frac{i}{2} \int d^{3} x \partial_{k} \chi^{T} \gamma^{k m} \dot{\mu}_{m}+\frac{i}{2} \int d^{3} x \mu_{k}^{T} \gamma^{k m} \partial_{m} \dot{\chi},
$$

which is formally logarithmically divergent; and

$$
\frac{i}{2} \int d^{3} x \mu_{k}^{T} \gamma^{k m} \dot{\mu}_{m},
$$

which is finite.

We analyse in turn the two divergent pieces.

- The first term is equal to

$$
\frac{i}{2} \int d^{3} x \partial_{k}\left(\chi^{T} \gamma^{k m} \partial_{m} \dot{\chi}\right)
$$

and so is a surface term that can be removed by subtracting it through $B$ in (2.2).

- The second term can be transformed into

$$
i \int d^{3} x \dot{\chi}^{T} \gamma^{k m} \partial_{k} \mu_{m}+i \oint d^{2} S_{k} \chi^{T} \gamma^{k m} \dot{\mu}_{m},
$$


(using $a^{T} \gamma^{k m} b=b^{T} \gamma^{k m} a$ for anticommuting spinors) up to a total time derivative that can again be absorbed in $B$. The surface term in (3.15) is clearly finite while the bulk term is also finite since we assume that the constraint $\mathcal{S}$ decays as $\mathcal{O}\left(r^{-4}\right)$.

So, if we adjust properly the surface term $B$, we get for the kinetic term

$$
K=i \int d^{3} x \dot{\chi}^{T} \gamma^{k m} \partial_{k} \mu_{m}+\frac{i}{2} \int d^{3} x \mu_{k}^{T} \gamma^{k m} \dot{\mu}_{m}+i \oint d^{2} S_{k} \chi^{T} \gamma^{k m} \dot{\mu}_{m}+B^{\prime},
$$

which is finite. The term $B^{\prime}$ is the undetermined left-over piece from $B$ that remains after the appropriate subtractions making the action finite have been performed. Space covariance and convergence requirements show that $B^{\prime}$ should be proportional to $i \oint d^{2} S_{k} \chi^{T} \gamma^{k m} \dot{\mu}_{m}$ and so henceforth we consider the most general kinetic term

$$
K=i \int d^{3} x \dot{\chi}^{T} \gamma^{k m} \partial_{k} \mu_{m}+\frac{i}{2} \int d^{3} x \mu_{k}^{T} \gamma^{k m} \dot{\mu}_{m}+\frac{i}{2} \alpha \oint d^{2} S_{k} \chi^{T} \gamma^{k m} \dot{\mu}_{m}
$$

where $\alpha$ is assumed to be a non-zero arbitrary constant. Note that for $\alpha=2$ one has that $B^{\prime}=0$. We also note that the fields $\chi$ and $\mu_{k}$ enter separately in the action and not only through the sum $\psi_{p}$, specifically in the kinetic term.

\subsection{Equations of motion}

We now verify that the action has a true extremum with our boundary conditions, and not just an extremum up to surface terms. The action for the generalized boundary conditions reads as above

$$
S=\int d t\left[K-i \int d^{3} x\left(\frac{1}{2} \mu_{k}^{T} \gamma_{0} \gamma^{k m n} \partial_{m} \mu_{n}+\psi_{0}^{T} \mathcal{S}\right)\right]
$$

where the kinetic term $K$ is now given by

$$
K=i \int d^{3} x\left(\dot{\chi}^{T} \gamma^{m n} \partial_{m} \mu_{n}+\frac{1}{2} \mu_{m}^{T} \gamma^{m n} \dot{\mu}_{n}\right)+\frac{i}{2} \alpha \oint d^{2} S_{m} \chi^{T} \gamma^{m n} \dot{\mu}_{n}
$$

We shall impose the same convenient asymptotic condition

$$
\psi_{0}=\mathcal{O}\left(\frac{1}{r^{2}}\right)
$$

on the Lagrange multiplier $\psi_{0}$.

The variation of (3.18) with respect to $\psi_{0}$ leads to the fermionic constraint

$$
\mathcal{S}=\gamma^{m n} \partial_{m} \mu_{n}=0 .
$$

The variation of the action with respect to $\mu_{m}$ turns out to be

$$
\begin{aligned}
\delta S=i \int d t\left\{\int d ^ { 3 } x \delta \mu _ { m } ^ { T } \left[\gamma ^ { m n } \left(\dot{\mu}_{n}+\partial_{n} \dot{\chi}-\right.\right.\right. & \left.\left.\partial_{n} \psi_{0}\right)-\gamma_{0} \gamma^{m n p} \partial_{n} \mu_{p}\right] \\
& \left.+\left(1-\frac{\alpha}{2}\right) \oint d^{2} S_{m} \delta \mu_{n}^{T} \gamma^{m n} \dot{\chi}\right\}
\end{aligned}
$$


where the boundary conditions have been taken into account to get rid of some boundary terms. If one imposes $\delta S=0$, the bulk contribution and the surface term must separately vanish. Vanishing of the bulk term reads

$$
\gamma^{m n}\left(\dot{\mu}_{n}+\partial_{n} \dot{\chi}-\partial_{n} \psi_{0}\right)-\gamma_{0} \gamma^{m n p} \partial_{n} \mu_{p}=0 .
$$

The leading order $1 / r$ of (3.23) reduces to the condition

$$
\gamma^{m k} \partial_{k} \dot{\bar{\chi}}=0
$$

on the leading term $\bar{\chi}$ in the asymptotic expansion of $\chi$, from which one infers

$$
\partial_{t}\left(\partial_{k} \bar{\chi}\right)=0 .
$$

The zero mode of $\bar{\chi}$ is thus an arbitrary function of time, but its higher spherical harmonic components are constant in time. The subsequent orders in (3.23) are just the standard dynamical Rarita-Schwinger equations for the gravitino field. The boundary term in (3.22) then vanishes as a direct consequence of (3.25) and the constraint (3.21) (for the zero mode).

The variation of (3.18) with respect to $\chi$ yields

$$
\delta S=\frac{i}{2} \alpha \oint d^{2} S_{m} \delta \chi^{T} \gamma^{m n} \dot{\mu}_{n}
$$

modulo the constraint. The above implies the following boundary equation of motion ${ }^{2}$

$$
\gamma^{r A} \dot{\bar{\mu}}_{A}=0
$$

for the angular component of $\mu_{k}$. Note that the next-to-leading order $\left(1 / r^{2}\right)$ of $(3.23)$ implies

$$
\gamma^{r A}\left(\dot{\bar{\mu}}_{A}+\bar{D}_{A} \dot{\chi}^{(1)}\right)=0
$$

where $\chi^{(1)}$ is the coefficient of the $(1 / r)$-term in the expansion of $\chi$. This leads to the Dirac equation on the 2 -sphere for $\dot{\chi}^{(1)}$, from which one also gets that the zero mode of $\chi^{(1)}$ is an arbitrary function of time, but its higher spherical harmonic components are constant in time.

We can thus conclude that the action (3.18) is a true extremum on the classical histories, which obey the Rarita-Schwinger equations of motion supplemented by (compatible) dynamical equations on the first terms in the development of $\chi$.

\subsection{Pre-symplectic form}

In order to discuss the canonical implementation of the Poincaré symmetry, we first need to understand the Poisson bracket structure of the theory.

The kinetic term (3.17) in the action yields the pre-symplectic form

$$
\begin{aligned}
\Omega= & -i \int d^{3} x d_{V} \chi^{T} \gamma^{k m} \partial_{k} d_{V} \mu_{m}+\frac{i}{2} \int d^{3} x d_{V} \mu_{k}^{T} \gamma^{k m} d_{V} \mu_{m} \\
& +\frac{i}{2} \alpha \oint d^{2} S_{k} d_{V} \chi^{T} \gamma^{k m} d_{V} \mu_{m} .
\end{aligned}
$$

\footnotetext{
${ }^{2}$ Here, $x^{A}$ denotes the coordinates on the 2 -sphere at infinity. In spherical coordinates, for example, $x^{A}=(\theta, \varphi)$ labels the two angles on the sphere.
} 
Note that since $\chi$ and $\mu_{m}$ are anticommuting, the one-forms $d_{V} \chi$ and $d_{V} \mu_{m}$ are commuting. In particular, $d_{V} \mu_{k}^{T} \gamma^{k m} d_{V} \mu_{m}$ is not zero. If the one-form $a$ is commuting, then $d_{V}(a \wedge b)=$ $d_{V} a \wedge b+a \wedge d_{V} b$.

This closed 2-form $\Omega$ form is degenerate, i.e., there exists a non-vanishing $Y$ such that $\iota_{Y} \Omega=0$. Our goal in this subsection is to determine all the null vectors $Y$ of $\Omega$.

The equation $\iota_{Y} \Omega=0$ reads

$$
\begin{aligned}
0= & -i \int d^{3} x \Upsilon^{T} \gamma^{k m} \partial_{k} d_{V} \mu_{m}-i \int d^{3} x d_{V} \chi^{T} \gamma^{k m} \partial_{k} \Sigma_{m} \\
& +\frac{i}{2} \int d^{3} x \Sigma_{k}^{T} \gamma^{k m} d_{V} \mu_{m}+\frac{i}{2} \int d^{3} x d_{V} \mu_{k}^{T} \gamma^{k m} \Sigma_{m} \\
& +\frac{i}{2} \alpha \oint d^{2} S_{k} \Upsilon^{T} \gamma^{k m} d_{V} \mu_{m}+\frac{i}{2} \alpha \oint d^{2} S_{k} d_{V} \chi^{T} \gamma^{k m} \Sigma_{m},
\end{aligned}
$$

with

$$
\delta_{Y} \chi=\Upsilon, \quad \delta_{Y} \mu_{m}=\Sigma_{m} .
$$

The terms involving $d_{V} \chi$ and $d_{V} \mu_{m}$ are independent and must vanish separately. Thus, one must have

$$
0=-i \int d^{3} x \Upsilon^{T} \gamma^{k m} \partial_{k} d_{V} \mu_{m}+i \int d^{3} x \Sigma_{k}^{T} \gamma^{k m} d_{V} \mu_{m}+\frac{i}{2} \alpha \oint d^{2} S_{k} \Upsilon^{T} \gamma^{k m} d_{V} \mu_{m},
$$

and

$$
0=-i \int d^{3} x d_{V} \chi^{T} \gamma^{k m} \partial_{k} \Sigma_{m}+\frac{i}{2} \alpha \oint d^{2} S_{k} d_{V} \chi^{T} \gamma^{k m} \Sigma_{m}
$$

The first line can be transformed into

$$
0=i \int d^{3} x\left(\partial_{k} \Upsilon^{T}+\Sigma_{k}^{T}\right) \gamma^{k m} d_{V} \mu_{m}-i\left(1-\frac{\alpha}{2}\right) \oint d^{2} S_{k} \Upsilon^{T} \gamma^{k m} d_{V} \mu_{m}
$$

which will be zero for arbitrary $d_{V} \mu_{m}$ satisfying the boundary conditions if the bulk and surface terms separately vanish. Vanishing of the bulk term imposes $\Sigma_{k}=-\partial_{k} \Upsilon$ from which it follows in particular that the leading term $\bar{\Upsilon}$ in $\Upsilon$ is constant since $\Sigma_{k}=\mathcal{O}\left(r^{-2}\right)$, i.e., $\Upsilon=C+\frac{\Upsilon^{(1)}\left(x^{A}\right)}{r}+\mathcal{O}\left(r^{-2}\right)$. The constant $C$ is unrestricted if $\alpha=2$ but must vanish for the surface term involving $d_{V} \mu_{m}$ to be zero when $\alpha \neq 2$.

Vanishing of the bulk term in the second equation (3.33) is an immediate consequence of $\Sigma_{k}=-\partial_{k} \Upsilon$, and vanishing of its surface term implies that $\Upsilon^{(1)}$ is also a constant $(\alpha \neq 0)$, so that we get as zero vector fields $Y$ of $\Omega$

$$
\Upsilon=C+\frac{C^{(1)}}{r}+\mathcal{O}\left(r^{-2}\right), \quad \Sigma_{k}=-\partial_{k} \Upsilon, \quad(\alpha=2)
$$

(and $C=0$ if $\alpha \neq 2$ ). When the pre-symplectic form is degenerate, the correspondence between phase space Hamiltonian vector fields and phase space functions is amended in an obvious way. A phase space vector field $X$ is still called Hamiltonian if $\mathcal{L}_{X} \Omega=0$, which is equivalent to $d_{V}\left(\iota_{X} \Omega\right)=0$. This implies $\iota_{X} \Omega=-d_{V} F$ for some function $F$ that is defined up to a constant and that is necessarily constant on the null submanifolds generated by the null vectors $Y$, since $0=\iota_{Y} d_{V} F=\mathcal{L}_{Y} F$. Conversely, a phase space function $F$ defines 
a Hamiltonian vector field only if it is constant on the null submanifolds generated by the null vectors $Y$, and the corresponding Hamiltonian vector field is then defined only up to a combination of the $Y$ 's. A recent instance where such a phenomenon occurs in an asymptotic analysis was studied in [47].

One can get a true symplectic structure by factoring out the null leaves of the presymplectic form. This can be done by imposing a gauge condition that freezes the possibility to move along the null leaves. One way to achieve this in our case is to impose that $\chi$ reduces to its first two terms in the asymptotic expansion, and that the coefficients $\bar{\chi}$ and $\chi^{(1)}$ have no zero mode.

\subsection{Canonical realization of the boosts - Need for a new surface field}

It is straightforward to check that the pre-symplectic form is invariant under spatial rotations and translations, given by (3.6) and (3.7) with $\xi=a^{\perp}$ and $\xi^{i}=b^{i}{ }_{j} x^{j}+a^{i}$, so that these transformations are canonical by construction with a well-defined generator. The situation is more complicated for boosts, for which

$$
\delta_{\xi} \chi=-\xi \gamma_{0} \gamma^{m} \partial_{m} \chi+\frac{1}{2} \partial_{j} \xi \gamma^{j} \gamma_{0} \chi, \quad \delta_{\xi} \mu_{p}=\xi \gamma_{p}{ }^{j k} \gamma_{0} \partial_{j} \mu_{k}+\frac{1}{2} \partial_{j} \xi \gamma^{j} \gamma_{0} \mu_{p}-\frac{1}{2} \xi \gamma_{p} \gamma_{0} \gamma^{j k} \partial_{j} \mu_{k},
$$

with

$$
\xi=b_{i} x^{i}, \quad\left(\xi^{k}=0\right) .
$$

The pre-symplectic form changes (off-shell) by the following surface term

$$
d_{V}\left(\iota_{\xi} \Omega\right)=\frac{i}{2} \oint d^{2} S_{k} \xi d_{V} \mu_{m}^{T} \gamma^{k m n} \gamma_{0} d_{V} \mu_{n}+\frac{i}{2} \alpha \oint d^{2} S_{k} \xi \partial_{m} d_{V} \chi^{T} \gamma_{0} \gamma^{k} d_{V} \mu^{m} .
$$

The first term vanishes once the boundary conditions are taken into account, while the second term is finite. The variation of the symplectic form then becomes

$$
d_{V}\left(\iota_{\xi} \Omega\right)=\frac{i}{2} \alpha \oint d^{2} S_{k} \xi \partial_{m} d_{V} \chi^{T} \gamma_{0} \gamma^{k} d_{V} \mu^{m}
$$

and is not zero, even if one takes into account that the constraints hold asymptotically.

In order for the boosts to be canonical transformations, we must find a way to get an invariant pre-symplectic form

$$
d_{V}\left(\iota_{\xi} \Omega\right)=0 \Rightarrow \iota_{\xi} \Omega=-d_{V} P_{\xi},
$$

which would allow us to define a canonical generator $P_{\xi}$ for boosts. To that end, we extend the phase space by adding new boundary degrees of freedom, the variation of which compensates the non-vanishing of (3.39).

Integrability of the boost generator. The non-vanishing of the right hand side of equation (3.39) is due to the presence of the field $\chi$. This problem does not arise in the supergravity analysis in [15], where the boost generator for the spin-3/2 field is immediately integrable, as there is no $\chi$ field in that construction. However, keeping the field $\chi$ is crucial in our case in order to obtain an infinite-dimensional set of improper gauge symmetries at 
spatial infinity. In analogy with the case of electromagnetism [36], we cure the problem by introducing an additional boundary field in the action principle to make the symplectic form invariant under boosts.

More specifically, we consider a vector-spinor field $\bar{\rho}^{k}$ depending only on the angles of the 2-sphere "at infinity", which extends into the bulk as

$$
\rho^{k}=\frac{\bar{\rho}^{k}}{r^{2}}+\mathcal{O}\left(\frac{1}{r^{3}}\right)
$$

endowed with the following symplectic form

$$
\Omega_{0}=-\frac{i}{2} \alpha \oint d^{2} S_{k} d_{V} \chi^{T} \gamma_{0} d_{V} \rho^{k} .
$$

We postulate the following infinitesimal transformation law under the Poincaré group,

$$
\delta_{b, Y} \bar{\rho}^{r}=-\gamma^{r} \bar{D}_{A}\left(b \bar{\mu}^{A}\right)-\frac{1}{2} b \bar{\gamma}_{A} \bar{\mu}^{A}+b \gamma_{0} \bar{\gamma}^{A} \bar{D}_{A} \bar{\rho}^{r}+\frac{3}{2} b \gamma^{r} \gamma_{0} \bar{\rho}^{r}-\frac{1}{2} \partial_{A} b \bar{\gamma}^{A} \gamma_{0} \bar{\rho}^{r}+\mathcal{L}_{Y} \bar{\rho}^{r}
$$

and for the angular components $\delta_{b, Y} \bar{\rho}^{A}=0$. (See appendix B for spherical coordinates). The change of the symplectic form (3.42) is given by

$$
d_{V}\left(\iota_{\xi} \Omega_{0}\right)=-\frac{i}{2} \alpha \oint d^{2} S_{k}\left(d_{V} \delta_{\xi} \chi^{T} \gamma_{0} d_{V} \rho^{k}+d_{V} \chi^{T} \gamma_{0} d_{V} \delta_{\xi} \rho^{k}\right) .
$$

For the boosts, this expression becomes, up to a total derivative on the 2-sphere,

$$
d_{V}\left(\iota_{\xi} \Omega_{0}\right)=-\frac{i}{2} \alpha \oint d^{2} S_{k} \xi \partial_{m} d_{V} \chi^{T} \gamma_{0} \gamma^{k} d_{V} \mu^{m} .
$$

Therefore, the total change of the symplectic form under boosts vanishes

$$
d_{V}\left(\iota_{\xi} \Omega\right)+d_{V}\left(\iota_{\xi} \Omega_{0}\right)=0
$$

which makes the boosts canonical transformations.

The introduction of the field $\rho^{k}$ modifies the kinetic term by a boundary contribution which one easily reads from $\Omega_{0}$, leading to

$$
K=i \int d^{3} x\left(\dot{\chi}^{T} \gamma^{m n} \partial_{m} \mu_{n}+\frac{1}{2} \mu_{m}^{T} \gamma^{m n} \dot{\mu}_{n}\right)+\frac{i}{2} \alpha \oint d^{2} S_{m}\left(\chi^{T} \gamma^{m n} \dot{\mu}_{n}-\chi^{T} \gamma_{0} \dot{\rho}^{m}\right) .
$$

The action principle with the additional boundary field now reads

$$
S=\int d t\left[K-i \int d^{3} x\left(\frac{1}{2} \mu_{k}^{T} \gamma_{0} \gamma^{k m n} \partial_{m} \mu_{n}+\psi_{0}^{T} \gamma^{m n} \partial_{m} \mu_{n}\right)\right] .
$$

It is easy to see that the action is invariant under arbitrary shifts of $\rho^{m}$,

$$
\delta_{\sigma} \rho^{m}=\sigma^{m}, \quad \delta_{\sigma} \chi=0, \quad \delta_{\sigma} \mu_{m}=0,
$$

where the parameter $\sigma^{m}$ falls off as

$$
\sigma_{m}=\frac{\bar{\sigma}_{m}}{r^{2}}+\mathcal{O}\left(\frac{1}{r^{3}}\right)
$$


It is useful to make the orthogonal decomposition of $\bar{\sigma}_{m}$ as

$$
\bar{\sigma}^{m}=\bar{\sigma} n^{m}+\bar{\theta}^{m}
$$

where $n^{m}=\frac{\partial}{\partial r}$ is normal to the sphere and $\bar{\theta}_{m} n^{m}=0$. Invariance of the action forces $\bar{\sigma}$ to be time-independent but imposes no condition on $\bar{\theta}^{m}$ because only the radial component $\bar{\rho}^{r}$ of $\bar{\rho}^{m}$ appears in the action. The angular components of $\bar{\rho}^{m}$ are absent and hence pure gauge degrees of freedom that can be "gauged away" (in fact they are already "away"!). The transformations parametrized by $\bar{\theta}_{m}$ are proper gauge transformations and there is only one degree of freedom in $\bar{\rho}^{m}$, namely, its radial component $\bar{\rho}^{r}$.

The change of variables $\rho^{m} \rightarrow \omega^{m}$ with

$$
\omega^{m}=\rho^{m}+\gamma_{0} \gamma^{m n} \mu_{n} \quad \Rightarrow \quad \bar{\omega}^{m}=\bar{\rho}^{m}+\gamma_{0} \gamma^{m n} \bar{\mu}_{n}
$$

brings the kinetic term $K$ to the simpler form

$$
K=i \int d^{3} x\left(\dot{\chi}^{T} \gamma^{m n} \partial_{m} \mu_{n}+\frac{1}{2} \mu_{m}^{T} \gamma^{m n} \dot{\mu}_{n}\right)-\frac{i}{2} \alpha \oint d^{2} S_{m} \chi^{T} \gamma_{0} \dot{\omega}^{m},
$$

which clearly involves only the radial component $\bar{\omega}^{r}$. Henceforth, we keep the formulations both in terms of $\rho^{k}$ and $\omega^{k}$, as they enlighten different aspects of the theory.

Pre-symplectic structure. Given the boundary modification of the action, the presymplectic form becomes

$$
\begin{aligned}
\Omega= & -i \int d^{3} x d_{V} \chi^{T} \gamma^{k m} \partial_{k} d_{V} \mu_{m}+\frac{i}{2} \int d^{3} x d_{V} \mu_{k}^{T} \gamma^{k m} d_{V} \mu_{m} \\
& +\frac{i}{2} \alpha \oint d^{2} S_{k} d_{V} \chi^{T} \gamma^{k m} d_{V} \mu_{m}-\frac{i}{2} \alpha \oint d^{2} S_{k} d_{V} \chi^{T} \gamma_{0} d_{V} \rho^{k}
\end{aligned}
$$

Note that in terms of $\omega^{k}$, the surface terms are combined into a single term:

$$
-\frac{i}{2} \alpha \oint d^{2} S_{k} d_{V} \chi^{T} \gamma_{0} d_{V} \omega^{k}
$$

The null vectors $Y$ are accordingly given by new expressions that are easily worked out. A direct computation shows that these are now (with $\delta_{Y} \chi=\Upsilon, \delta_{Y} \mu_{m}=\Sigma_{m}$ and $\delta_{Y} \rho^{k}=R^{k}$ ),

$$
\Upsilon=\frac{C^{(1)}}{r}+\mathcal{O}\left(r^{-2}\right), \quad \Sigma_{k}=-\partial_{k} \Upsilon, \quad R^{r}=\gamma^{r A} \partial_{A} \Upsilon, \quad R^{A}=y^{A},
$$

where $y^{A}$ are arbitrary functions. In particular, the zero mode of $\bar{\chi}$ is not pure gauge anymore since the constant $C$ of (3.35) is now forced to be equal to zero in (3.56) even when $\alpha=2$. This is because the zero mode of $\rho^{r}$ is present in the new action.

Equations of motion. The boundary modification of the action leads also to a slight change in the boundary equations of motion.

Variation of the complete action (3.48) with respect to $\rho^{k}$ yields $\dot{\bar{\chi}}=0$. This boundary equation of motion is a consequence of the unchanged equations of motion obtained by 
varying $\mu_{k}$, with the only additional information that the zero mode of $\bar{\chi}$ should also be constant in time (instead of being arbitrary).

If one varies the complete action (3.48) with respect to $\chi$, one gets

$$
\delta S=\frac{i}{2} \alpha \oint d^{2} S_{m} \delta \chi^{T}\left(\gamma^{m n} \dot{\mu}_{n}-\gamma_{0} \dot{\rho}^{m}\right),
$$

modulo the constraint. The boundary equation of motion is thus now given by

$$
\gamma^{r A} \dot{\bar{\mu}}_{A}-\gamma_{0} \dot{\bar{\rho}}^{r}=0 \quad \Leftrightarrow \quad \dot{\bar{\omega}}^{r}=0,
$$

an equation that can be viewed as fixing $\dot{\bar{\rho}}$ in terms of $\dot{\bar{\mu}}_{A}$.

\subsection{Poincaré generators}

The addition of the new boundary field renders all the Poincaré transformations canonical, and consequently leads to well-defined generators through its relation with the symplectic form

$$
\iota_{X} \Omega=-d_{V} G .
$$

With the expression of the infinitesimal Poincaré transformations of the fields written above,

$$
\begin{aligned}
\delta_{\xi} \chi & =-\xi \gamma_{0} \gamma^{m} \partial_{m} \chi+\frac{1}{2} \partial_{j} \xi \gamma^{j} \gamma_{0} \chi+\mathcal{L}_{\xi} \chi \\
\delta_{\xi} \mu_{p} & =\xi \gamma_{p}^{j k} \gamma_{0} \partial_{j} \mu_{k}+\frac{1}{2} \partial_{j} \xi \gamma^{j} \gamma_{0} \mu_{p}-\frac{1}{2} \xi \gamma_{p} \gamma_{0} \gamma^{j k} \partial_{j} \mu_{k}+\mathcal{L}_{\xi} \mu_{p} \\
\delta_{b, Y} \bar{\rho}^{r} & =-\gamma^{r} \bar{D}_{A}\left(b \bar{\mu}^{A}\right)-\frac{1}{2} b \bar{\gamma}_{A} \bar{\mu}^{A}+b \gamma_{0} \bar{\gamma}^{A} \bar{D}_{A} \bar{\rho}^{r}+\frac{3}{2} b \gamma^{r} \gamma_{0} \bar{\rho}^{r}-\frac{1}{2} \partial_{A} b \bar{\gamma}^{A} \gamma_{0} \bar{\rho}^{r}+\mathcal{L}_{Y} \bar{\rho}^{r} \\
& \Leftrightarrow \quad \delta_{b, Y} \bar{\omega}^{r}=b \gamma_{0} \bar{\gamma}^{A} \bar{D}_{A} \bar{\omega}^{r}+\frac{3}{2} b \gamma^{r} \gamma_{0} \bar{\omega}^{r}-\frac{1}{2} \partial_{A} b \bar{\gamma}^{A} \gamma_{0} \bar{\omega}^{r}+\mathcal{L}_{Y} \bar{\omega}^{r}
\end{aligned}
$$

one finds that the Poincaré generators are explicitly given by

$$
\begin{aligned}
P_{\xi, \xi^{i}}= & \int d^{3} x\left(\xi \mathcal{H}^{\mathrm{RS}}+\xi^{i} \mathcal{H}_{i}^{\mathrm{RS}}\right)+\mathcal{B}_{\xi, \xi^{i}}^{\mathrm{RS}}, \\
\mathcal{H}^{\mathrm{RS}}= & \frac{i}{2}\left[\mu_{k}^{T} \gamma^{k m n} \gamma_{0} \partial_{m} \mu_{n}+\partial_{k}\left(\mu_{j}^{T} \gamma^{j} \gamma_{0} \mu^{k}\right)+\partial_{k}\left(\chi^{T} \gamma^{k} \gamma_{0} \gamma^{m n} \partial_{m} \mu_{n}\right)\right. \\
& \left.+2 \partial_{k} \chi^{T} \gamma_{0} \gamma^{k} \gamma^{p q} \partial_{p} \mu_{q}\right], \\
\mathcal{H}_{i}^{\mathrm{RS}}= & -i\left[\frac{1}{2} \partial_{m}\left(\mu_{n}^{T} \gamma^{m n} \mu_{k}\right)+\frac{1}{2} \mu_{m}^{T} \gamma^{m n} \partial_{k} \mu_{n}+\frac{1}{8} \partial_{p}\left(\mu_{m}^{T} \gamma^{m n} \gamma_{k}^{p} \mu_{n}\right)\right. \\
& \left.+\partial_{k} \chi^{T} \gamma^{m n} \partial_{m} \mu_{n}+\frac{1}{4} \partial_{p}\left(\chi^{T} \gamma_{k}^{p} \gamma^{m n} \partial_{m} \mu_{n}\right)\right], \\
\mathcal{B}_{\xi, \xi^{i}}^{\mathrm{RS}}= & \frac{i}{2} \alpha \oint d^{2} S_{k}\left(\delta_{\xi} \chi^{T} \gamma_{0} \omega^{k}+\mathcal{L}_{\xi_{i}} \chi^{T} \gamma_{0} \omega^{k}\right) .
\end{aligned}
$$

where $\omega^{k}$ is defined in (3.52). In the $\omega$-formulation, $\mathcal{B}_{\xi, \xi^{i}}^{\mathrm{RS}}$ gets simplified to

$$
\frac{i}{4} \alpha \oint d^{2} S_{k}\left[\partial_{j} \xi \chi^{T} \gamma^{j} \omega^{k}-2 \xi^{n} \partial_{n} \chi^{T} \gamma_{0} \omega^{k}+\frac{1}{2} \partial_{p} \xi_{q} \chi^{T} \gamma^{p q} \gamma_{0} \omega^{k}\right] .
$$




\subsection{A twofold of infinite-dimensional fermionic improper gauge symmetries}

Similarly, the canonical generator of the proper and improper gauge symmetries reads

$$
\begin{aligned}
G_{\epsilon, \varepsilon, \sigma^{k}}^{\mathrm{RS}}= & i \int d^{3} x\left(\epsilon^{T}+\varepsilon^{T}\right) \gamma^{k m} \partial_{k} \mu_{m} \\
& +\frac{i}{2} \alpha \oint d^{2} S_{k}\left(\epsilon^{T} \gamma^{k m} \mu_{m}-\chi^{T} \gamma^{k m} \partial_{m} \varepsilon-\epsilon^{T} \gamma_{0} \rho^{k}+\chi^{T} \gamma_{0} \sigma^{k}\right),
\end{aligned}
$$

generating the infinitesimal transformation laws of the fields

$$
\begin{aligned}
\delta_{\epsilon} \chi & =\epsilon, \\
\delta_{\varepsilon} \mu_{m} & =\partial_{m} \varepsilon, \\
\delta_{\sigma_{m}} \rho^{m} & =\sigma^{m} .
\end{aligned}
$$

One can rewrite the generator (3.68) in terms of the new variable $\omega^{k}$ as

$$
\begin{aligned}
G_{\epsilon, \varepsilon, \sigma^{k}}^{\mathrm{RS}} & \equiv G_{\epsilon, \varepsilon, \zeta^{k}}^{\mathrm{RS}} \\
& =i \int d^{3} x\left(\epsilon^{T}+\varepsilon^{T}\right) \gamma^{k m} \partial_{k} \mu_{m}+\frac{i}{2} \alpha \oint d^{2} S_{k}\left(\chi^{T} \gamma_{0} \zeta^{k}-\epsilon^{T} \gamma_{0} \omega^{k}\right),
\end{aligned}
$$

where we have set $\zeta^{k}=\sigma^{k}+\gamma_{0} \gamma^{k m} \partial_{m} \varepsilon$, so that the transformations of the field $\omega^{k}$ read

$$
\delta_{\zeta_{m}} \omega^{m}=\zeta^{m} .
$$

The bulk term of the charge (3.72) is proportional to the constraint. The surface integral to which the charge reduces on-shell does not vanish if the leading terms $\bar{\epsilon}$ and $\bar{\zeta}^{r}$ in the expansions of $\epsilon$ and $\zeta^{r}$ are not zero. These are the improper gauge symmetries. By contrast, the transformations with $\varepsilon \neq 0$ but $\bar{\epsilon}=\bar{\zeta}^{r}=0$ are proper gauge transformations. Thus, the symmetry turns out to be two-fold (if $\alpha \neq 0$ ), labelled by the leading order parameters $\bar{\epsilon}$ and $\bar{\zeta}^{r}$.

Using the proper gauge transformations, one can force the field $\chi$ to reduce to its leading term,

$$
\chi=\bar{\chi}
$$

which justifies why it is called a surface field.

It is curious that the bulk term of the gauge transformations with $\epsilon=-\varepsilon$ identically vanishes. These are improper when $\bar{\zeta}^{r} \neq 0$. Thus, there exist improper gauge transformations that are generated by pure boundary terms. This is possible because the (pre)symplectic form involves non trivial surface contributions, as it happens in the case of the duality-invariant formulation of electromagnetism [47].

We shall use both parametrizations $G_{\epsilon, \varepsilon, \sigma^{k}}^{\mathrm{RS}}$ and $G_{\epsilon, \varepsilon, \zeta^{k}}^{\mathrm{RS}}$ of the gauge generators. The first one is the natural one when one starts from the Rarita-Schwinger formulation of the action, as we have done here. The second one is more adapted to the description of the independent improper gauge symmetries, since these are just characterized by the simple conditions $\bar{\epsilon}=0, \bar{\zeta}^{r}=0$, which take a "non-diagonal form" that mixes the parameters in the other parametrization. 


\subsection{Asymptotic symmetry algebra}

The bracket algebra of the canonical generators of gauge and Poincaré symmetries reads

$$
\begin{aligned}
\left\{P_{\xi_{1}, \xi_{1}^{i}}, P_{\xi_{2}, \xi_{2}^{i}}\right\} & =P_{\hat{\xi}, \hat{\xi}^{i}}, \\
\left\{P_{\xi, \xi^{i}}, G_{\epsilon, \varepsilon, \sigma^{k}}^{\mathrm{RS}}\right\} & =G_{\hat{\epsilon}, \hat{\varepsilon}, \hat{\sigma}^{k}}^{\mathrm{RS}}, \\
\left\{G_{\tau_{1}}^{\mathrm{RS}}, G_{\tau_{2}}^{\mathrm{RS}}\right\} & =\mathcal{C}_{\left\{\tau_{1}, \tau_{2}\right\}},
\end{aligned}
$$

where $\tau_{i}=\left\{\epsilon_{i}, \varepsilon_{i}, \sigma_{i}^{k}\right\}$ with $i=1,2$, and where the hatted parameters of the commutator transformations are given by

$$
\begin{aligned}
\hat{\xi} & =\xi_{1}^{i} \partial_{i} \xi_{2}-\xi_{2}^{i} \partial_{i} \xi_{1}, \\
\hat{\xi}^{i} & =\xi_{1}^{j} \partial_{j} \xi_{2}^{i}-\xi_{2}^{j} \partial_{j} \xi_{1}^{i}+\xi_{1} \partial^{i} \xi_{2}-\xi_{2} \partial^{i} \xi_{1}, \\
\hat{\epsilon} & =-\xi \gamma_{0} \gamma^{m} \partial_{m} \epsilon+\frac{1}{2} \partial_{j} \xi \gamma^{j} \gamma_{0} \epsilon+\mathcal{L}_{\xi} \epsilon \\
\hat{\varepsilon} & =-\xi \gamma_{0} \gamma^{m} \partial_{m} \varepsilon+\frac{1}{2} \partial_{m} \xi \gamma^{m} \gamma_{0} \varepsilon+\mathcal{L}_{\xi} \varepsilon, \\
\hat{\sigma}^{r} & =-\gamma^{r} \bar{D}_{A}\left(b \bar{D}^{A} \varepsilon\right)-\frac{1}{2} b \bar{\gamma}_{A} \bar{D}^{A} \varepsilon+b \gamma_{0} \bar{\gamma}^{A} \bar{D}_{A} \sigma^{r} \\
& +\frac{3}{2} b \gamma^{r} \gamma_{0} \sigma^{r}-\frac{1}{2} \partial_{A} b \bar{\gamma}^{A} \gamma_{0} \sigma^{r}+\mathcal{L}_{Y} \sigma^{r} .
\end{aligned}
$$

Only the asymptotic parts $\overline{\hat{\tau}}$ of the gauge parameters — the ones defining the improper transformations - are actually meaningful since one has always the freedom of adding proper gauge transformations to $\hat{\tau}$. This freedom can be frozen by fixing the gauge and using the Dirac bracket, but the meaningful, gauge invariant, asymptotic information on the algebra can already be extracted without going through this procedure.

The bracket algebra of the generators of the improper gauge transformations provides a projective representation of the algebra of the transformations themselves, with a central extension $\mathcal{C}_{\left\{\tau_{1}, \tau_{2}\right\}}$ that can be non trivial $[13,48]$. Here, non-trivial central charges are allowed because the fermionic improper gauge symmetries are abelian and indeed do occur. They are given by

$$
\begin{gathered}
\mathcal{C}_{\{\epsilon, \varepsilon\}}=-\mathcal{C}_{\{\varepsilon, \epsilon\}}=\frac{i}{2} \alpha \oint d^{2} S_{k} \epsilon^{T} \gamma^{k m} \partial_{m} \varepsilon, \\
\mathcal{C}_{\left\{\epsilon, \sigma^{k}\right\}}=-\mathcal{C}_{\left\{\sigma^{k}, \epsilon\right\}}=-\frac{i}{2} \alpha \oint d^{2} S_{k} \epsilon^{T} \gamma_{0} \sigma^{k} .
\end{gathered}
$$

A consistency check is that the central charge vanishes when one of the gauge transformations is a proper gauge symmetry, i.e., in the specific case considered here, $\zeta^{k}=$ $\sigma^{k}+\gamma_{0} \gamma^{k m} \partial_{m} \varepsilon=0$.

We close this section with two observations.

- The leading orders $\bar{\epsilon}, \bar{\varepsilon}, \bar{\sigma}^{r}$ of the gauge parameters, characterizing the improper gauge transformations, are functions on the 2-sphere transforming in infinite-dimensional representations of the Lorentz group that can be read off from (3.80)-(3.82). (Covariantly) Constant spinor fields define a four-dimensional irreducible representation, the "zero mode" representation. 
- To remove the zero mode of the fermionic improper gauge symmetry parametrized by $\bar{\epsilon}$, one could impose the transverse condition $\partial_{k} \rho^{k}=0$ on $\rho^{k}$. This is consistent with Lorentz invariance and makes the zero mode of $\chi$ pure gauge.

More on this will be discussed elsewhere.

\section{Rigid supersymmetry: spin- $\left(1, \frac{3}{2}\right)$ multiplet}

We shall now analyze the compatibility of the infinite-dimensional asymptotic symmetries with the super-Poincaré algebra in four dimensions. We start with the simplest case of the spin- $\left(1, \frac{3}{2}\right)$ multiplet under rigid supersymmetry, which corresponds to the sum of the free Maxwell and free Rarita-Schwinger actions

$$
S=S_{\mathrm{EM}}+S_{\mathrm{RS}}
$$

where [36]

$$
\begin{aligned}
S_{\mathrm{EM}}=\int d t\left\{\int\right. & d^{3} x\left(\pi^{i} \dot{A}_{i}+\pi_{\Psi} \dot{\Psi}\right)-\oint d^{2} S_{i} A^{i} \dot{\Psi} \\
& \left.-\int d^{3} x\left(\frac{1}{2} \pi^{i} \pi_{i}+\frac{1}{4} F^{i j} F_{i j}+\lambda \pi_{\Psi}-A_{t} \partial_{i} \pi^{i}\right)\right\},
\end{aligned}
$$

and the Rarita-Schwinger action $S_{\mathrm{RS}}$ is given in (3.48).

Under rigid supersymmetry the fields transform as

$$
\begin{aligned}
\delta_{\epsilon_{0}} A_{k} & =i \epsilon_{0}^{T} \gamma_{0} \partial_{k} \chi+i \epsilon_{0}^{T} \gamma_{0} \mu_{k}, \\
\delta_{\epsilon_{0}} \pi^{k} & =-i \epsilon^{k l m} \epsilon_{0}^{T} \gamma_{0} \gamma_{5} \partial_{l} \mu_{m}, \\
\delta_{\epsilon_{0}} \mu_{k} & =\frac{1}{2} \gamma_{m} \gamma_{k} W^{m} \gamma_{0} \epsilon_{0}, \\
\delta_{\epsilon_{0}} \chi & =0
\end{aligned}
$$

where $W^{k}=\pi^{k}-\epsilon^{k l m} \partial_{l} A_{m} \gamma_{5}$, and $\epsilon_{0}$ is a constant spinor parameter. These transformations leave the piece of the total action (4.1) involving only the fields $A_{k}, \pi^{k}, \mu_{k}$ and $\chi$ invariant up to non-vanishing boundary terms. We shall remedy this problem by defining the transformation laws of the boundary fields $\Psi$ and $\rho^{k}$ in $S$ so that the symplectic form and the action be invariant under rigid supersymmetry.

This will not be sufficient for fully solving the problem, and an extra step must be simultaneously taken. As all rigid symmetries are defined up to a gauge symmetry, we have the freedom to add a gauge transformation to the supersymmetry transformations denote it by $\nu$ - which can depend on the fields. The variation of the symplectic form under supersymmetry and the accompanying gauge transformation reads $d_{V}\left(\iota_{\epsilon_{0}} \Omega+\iota_{\nu} \Omega\right)$. It turns out that one cannot define the supersymmetry transformations of the boundary fields so that $d_{V}\left(\iota_{\epsilon_{0}} \Omega\right)$ vanishes by itself. It is also necessary to add a $\mathrm{U}(1)$ gauge transformation with a field dependent parameter

$$
\nu_{\left(\epsilon_{0}\right)} \equiv \mathcal{F}^{T} \epsilon_{0}, \quad\left(\Rightarrow \nu_{\left(\epsilon_{0}\right)}=0 \text { if } \epsilon_{0}=0\right),
$$


where $\mathcal{F}$ is a function of the fermionic fields specified below. Only then can one achieve

$$
d_{V}\left(\iota_{\epsilon_{0}} \Omega+\iota_{\nu} \Omega\right)=0
$$

a condition that is necessary for integrability of the supersymmetry charges.

\subsection{Invariance under rigid supersymmetry}

The symplectic form for the multiplet $\left(1, \frac{3}{2}\right)$ is explicitly given by

$$
\Omega=\Omega_{\mathrm{EM}}+\Omega_{\mathrm{RS}},
$$

where

$$
\begin{aligned}
\Omega_{\mathrm{EM}}= & \int d^{3} x\left(d_{V} \pi^{k} d_{V} A_{k}+d_{V} \pi_{\Psi} d_{V} \Psi\right)-\oint d^{2} S_{k} d_{V} A^{k} d_{V} \Psi \\
\Omega_{\mathrm{RS}}= & -i \int d^{3} x d_{V} \chi^{T} \gamma^{k m} \partial_{k} d_{V} \mu_{m}+\frac{i}{2} \int d^{3} x d_{V} \mu_{k}^{T} \gamma^{k m} d_{V} \mu_{m} \\
& +\frac{i}{2} \alpha \oint d^{2} S_{k}\left(d_{V} \chi^{T} \gamma^{k m} d_{V} \mu_{m}-d_{V} \chi^{T} \gamma_{0} d_{V} \rho^{k}\right) .
\end{aligned}
$$

Under rigid supersymmetry, it changes as

$$
\begin{aligned}
d_{V}\left(\iota_{\epsilon_{0}} \Omega\right)= & \int d^{3} x\left(d_{V} \delta_{\epsilon_{0}} \pi_{\Psi} d_{V} \Psi+d_{V} \pi_{\Psi} d_{V} \delta_{\epsilon_{0}} \Psi\right) \\
& +\oint d^{2} S_{m}\left(i d_{V} \pi^{m} d_{V} \chi^{T} \gamma_{0} \epsilon_{0}-d_{V} A^{m} d_{V} \delta_{\epsilon_{0}} \Psi\right) \\
& -\alpha i \oint d^{2} S_{m}\left(\frac{1}{2} d_{V} \chi^{T} d_{V} W^{m} \gamma_{0} \epsilon_{0}+d_{V} \chi^{T} \gamma_{0} d_{V} \delta_{\epsilon_{0}} \rho^{m}\right)
\end{aligned}
$$

The second term can be made zero by imposing the following transformation law for the boundary field $\rho^{m}$

$$
\delta_{\epsilon_{0}} \rho^{m}=\frac{1}{2} \gamma_{0} W^{m} \gamma_{0} \epsilon_{0} \quad \Leftrightarrow \quad \delta_{\epsilon_{0}} \omega^{m}=0 .
$$

The first term can be removed by applying the field dependent $\mathrm{U}(1)$ gauge transformation

$$
d_{V}\left(\iota_{\nu} \Omega\right)=i \oint d^{2} S_{m} d_{V} \pi^{m} d_{V} \nu
$$

with $\nu=\chi^{T} \gamma_{0} \epsilon_{0}$, and choosing $\delta_{\epsilon_{0}} \pi_{\Psi}=\delta_{\epsilon_{0}} \Psi=0$. We then obtain that

$$
d_{V}\left(\iota_{\epsilon_{0}} \Omega+\iota_{\nu} \Omega\right)=0
$$

\subsection{Canonical realization of the asymptotic symmetries}

Once supersymmetry is defined on the fields as above, the theory is invariant under the rigid super-Poincare transformations. It is also invariant under the improper gauge symmetries of the spin- 1 and spin- $\frac{3}{2}$ fields, which are unaffected by the construction. We examine their generators in turn. 


\subsubsection{Super-Poincaré algebra}

The canonical generators of Poincaré transformations are given by the sum

$$
P_{\xi, \xi^{i}}=P_{\xi, \xi^{i}}^{\mathrm{EM}}+P_{\xi, \xi^{i}}^{\mathrm{RS}}
$$

since the spin- 1 and spin- $\frac{3}{2}$ fields are uncoupled. The charges are given by

$$
\begin{aligned}
P_{\xi, \xi^{i}}^{\mathrm{EM}} & =\int d^{3} x\left(\xi \mathcal{H}^{\mathrm{EM}}+\xi^{i} \mathcal{H}_{i}^{\mathrm{EM}}\right)+\mathcal{B}_{\xi, \xi^{i}}^{\mathrm{EM}}, \\
\mathcal{H}^{\mathrm{EM}} & =-\Psi \partial_{i} \pi^{i}-A_{i} \partial^{i} \pi_{\Psi}+\frac{1}{2} \pi_{i} \pi^{i}+\frac{1}{4} F_{i j} F^{i j}, \\
\mathcal{H}_{i}^{\mathrm{EM}} & =F_{i j} \pi^{j}-A_{i} \partial_{j} \pi^{j}+\pi_{\Psi} \partial_{i} \Psi, \\
\mathcal{B}_{\xi, \xi^{i}}^{\mathrm{EM}} & =\oint d^{2} x\left[b\left(\bar{\Psi} \bar{\pi}^{r}+\sqrt{\bar{\gamma}} \bar{A}_{B} \bar{D}^{B} A_{r}\right)+Y^{B}\left(\bar{A}_{B} \bar{\pi}^{r}+\sqrt{\bar{\gamma}} \bar{\Psi} \partial_{B} \bar{A}_{r}\right)\right] .
\end{aligned}
$$

for the electromagnetic field $[32,36]$ and $P_{\xi, \xi^{i}}^{\mathrm{RS}}$ given in (3.63) for the Rarita-Schwinger field.

Infinitesimal Poincaré transformations for Maxwell fields read [32, 36]

$$
\begin{aligned}
\delta_{\xi} A_{i} & =\xi \pi_{i}+\mathcal{L}_{\xi} A_{i}, \\
\delta_{\xi} \pi^{i} & =\partial_{j}\left(\xi F^{j i}\right)+\mathcal{L}_{\xi} \pi^{i}, \\
\delta_{\xi} \Psi & =\partial_{i}\left(\xi A^{i}\right)+\xi^{i} \partial_{i} \Psi, \\
\delta_{\xi} \pi_{\Psi} & =\xi \partial_{i} \pi^{i}+\partial_{i}\left(\xi^{i} \pi_{\Psi}\right) \approx 0,
\end{aligned}
$$

and the ones for Rarita-Schwinger fields are given in (3.60), (3.61), (3.62).

The canonical generator of rigid supersymmetry follows from the relation $\iota_{\epsilon_{0}} \Omega+$ $\iota_{\nu\left(\epsilon_{0}\right)} \Omega=-d_{V} G_{\epsilon_{0}}^{\mathrm{SUSY}}$ and is given by

$$
G_{\epsilon_{0}}^{\mathrm{SUSY}}=i \int d^{3} x \mu_{k}^{T} W^{k} \gamma_{0} \epsilon_{0} .
$$

Infinitesimal rigid supersymmetry transformations of all the fields in the multiplet are then

$$
\begin{aligned}
\delta_{\epsilon_{0}} A_{k} & =i \epsilon_{0}^{T} \gamma_{0} \mu_{k}, \\
\delta_{\epsilon_{0}} \pi^{k} & =-i \epsilon^{k l m} \epsilon_{0}^{T} \gamma_{0} \gamma_{5} \partial_{l} \mu_{m}, \\
\delta_{\epsilon_{0}} \mu_{k} & =\frac{1}{2} \gamma_{m} \gamma_{k} W^{m} \gamma_{0} \epsilon_{0}, \\
\delta_{\epsilon_{0}} \rho^{m} & =\frac{1}{2} \gamma_{0} W^{m} \gamma_{0} \epsilon_{0} \\
\delta_{\epsilon_{0}} \Psi & =\delta_{\epsilon_{0}} \chi=0
\end{aligned}
$$

It is straightforward to verify that the super-Poincare algebra is satisfied

$$
\begin{aligned}
\left\{P_{\xi_{1}, \xi_{1}^{i}}, P_{\xi_{2}, \xi_{2}^{i}}\right\} & =P_{\hat{\xi}, \hat{\xi}^{i}}, \\
\left\{P_{\xi, \xi^{i}}, G_{\epsilon_{0}}^{\mathrm{SUSY}}\right\} & =G_{\hat{\epsilon}_{0}}^{\mathrm{SUSY}}, \\
\left\{G_{\epsilon_{0}^{1}}^{\mathrm{SUSY}}, G_{\epsilon_{0}^{2}}^{\mathrm{SUSY}}\right\} & =P_{\hat{a}, \hat{a}^{i}},
\end{aligned}
$$


where the hatted parameters of the commutator transformation are

$$
\begin{aligned}
\hat{\epsilon}_{0} & =\frac{1}{2} \partial_{m} \xi \gamma^{m} \gamma_{0} \epsilon_{0}+\mathcal{L}_{\xi} \epsilon_{0}, \\
\hat{a} & =\frac{i}{2}\left(\epsilon_{0}^{2}\right)^{T} \epsilon_{0}^{1}, \quad \hat{a}^{i}=-\frac{i}{2}\left(\epsilon_{0}^{2}\right)^{T} \gamma^{i} \gamma_{0} \epsilon_{0}^{1},
\end{aligned}
$$

with $\hat{\xi}$ and $\hat{\xi}^{i}$ given in (3.78) and (3.79), respectively.

It is worth noting that the bracket between two rigid supersymmetry generators (4.33) correctly closes to spatial and time translations, as expected for the canonical realization of the super-Poincaré algebra on the spin- $(1,3 / 2)$ multiplet.

\subsubsection{Infinite-dimensional algebra of improper gauge symmetries}

Since the two fields in the supermultiplet do not interact, the symmetry sector of the improper gauge symmetries is just the direct sum of the asymptotic angle-dependent $u(1)$ transformations of the Maxwell field and the asymptotic angle-dependent fermionic transformations of the Rarita-Schwinger field. The canonical generators of improper gauge symmetries are unchanged. For completeness, we reproduce them here, together with their algebra,

$$
\begin{aligned}
G_{\mu, \nu}^{\mathrm{EM}}= & \int d^{3} x\left(\mu \pi_{\Psi}-\nu \partial_{i} \pi^{i}\right)+\oint d^{2} S_{m}\left(\nu \pi^{m}-\mu A^{m}\right), \\
G_{\epsilon, \varepsilon, \sigma^{k}}^{\mathrm{RS}}= & i \int d^{3} x\left(\epsilon^{T}+\varepsilon^{T}\right) \gamma^{k m} \partial_{k} \mu_{m} \\
& +\frac{i}{2} \alpha \oint d^{2} S_{k}\left(\epsilon^{T} \gamma^{k m} \mu_{m}-\chi^{T} \gamma^{k m} \partial_{m} \varepsilon-\epsilon^{T} \gamma_{0} \rho^{k}+\chi^{T} \gamma_{0} \sigma^{k}\right) .
\end{aligned}
$$

Transformation laws for the Maxwell field under gauge symmetries read

$$
\delta_{\mu, \nu} \Psi=\mu, \quad \delta_{\mu, \nu} A_{i}=\partial_{i} \nu, \quad \delta_{\mu, \nu} \pi^{i}=\delta_{\mu, \nu} \pi_{\Psi}=0,
$$

while the ones for Rarita-Schwinger fields are given in (3.69), (3.70), (3.71).

The brackets of improper gauge generators with Poincaré read

$$
\begin{aligned}
\left\{P_{\xi, \xi^{i}}, G_{\mu, \nu}^{\mathrm{EM}}\right\} & =G_{\hat{\mu}, \hat{\nu}}^{\mathrm{EM}}, \\
\left\{P_{\xi, \xi^{i}}, G_{\epsilon, \varepsilon, \sigma^{k}}^{\mathrm{RS}}\right\} & =G_{\hat{\epsilon}, \hat{\varepsilon}, \hat{\sigma}^{k}}^{\mathrm{RS}},
\end{aligned}
$$

where the parameters transform as

$$
\hat{\nu}=\xi \mu+\mathcal{L}_{\xi} \rho, \quad \hat{\mu}=\partial^{m}\left(\xi \partial_{m} \nu\right)+\mathcal{L}_{\xi} \nu_{m},
$$

with $\hat{\epsilon}, \hat{\varepsilon}$ and $\hat{\sigma}$ given in (3.80), (3.81) and (3.82), respectively.

The brackets between improper gauge symmetries form a centrally extended Abelian gauge algebra

$$
\begin{aligned}
\left\{G_{\mu_{1}, \nu_{1}}^{\mathrm{EM}}, G_{\mu_{2}, \nu_{2}}^{\mathrm{EM}}\right. & =0, \\
\left\{G_{\mu, \nu}^{\mathrm{EM}}, G_{\tau}^{\mathrm{RS}}\right\} & =0, \\
\left\{G_{\tau_{1}}^{\mathrm{RS}}, G_{\tau_{2}}^{\mathrm{RS}}\right\} & =\mathcal{C}_{\left\{\tau_{1}, \tau_{2}\right\}},
\end{aligned}
$$

where $\tau_{i}=\left\{\epsilon_{i}, \varepsilon_{i}, \sigma_{i}^{k}\right\}$ with $i=1,2$, and $\mathcal{C}_{\left\{\tau_{1}, \tau_{2}\right\}}$ are given in (3.83) and (3.84). 


\section{Rigid supersymmetry: spin- $\left(2, \frac{3}{2}\right)$ multiplet}

We now turn to the spin- $\left(2, \frac{3}{2}\right)$ multiplet, which is the relevant one for analyzing the superBMS extensions in four dimensions. The procedure parallels the one we followed for the spin- $\left(1, \frac{3}{2}\right)$ multiplet.

The system is described by the sum of the free spin-2 (Pauli-Fierz theory) and free Rarita-Schwinger actions

$$
S=S_{\mathrm{PF}}+S_{\mathrm{RS}}
$$

The Hamiltonian action for the free spin-2 theory on a Minkowski background reads

$$
S_{\mathrm{PF}}=\int d t d^{3} x\left(\pi^{i j} \dot{h}_{i j}-\mathcal{E}-n \mathcal{G}-n^{i} \mathcal{G}_{i}\right)
$$

where the energy density and the Hamiltonian constraints associated to the Lagrange multipliers $n$ and $n^{i}$, are given by

$$
\begin{aligned}
\mathcal{E} & =\pi^{i j} \pi_{i j}-\frac{\pi^{2}}{2}+\frac{1}{4} \partial_{k} h_{i j} \partial^{k} h^{i j}-\frac{1}{2} \partial_{j} h^{i j} \partial^{k} h_{i k}+\frac{1}{4} \partial_{i} h \partial^{i} h+\frac{1}{2} h \mathcal{G}, \\
\mathcal{G} & =\triangle h-\partial_{i} \partial_{j} h^{i j}, \\
\mathcal{G}_{i} & =-2 \partial_{j} \pi_{i}^{j},
\end{aligned}
$$

respectively. This theory is left invariant by the following gauge transformations

$$
\begin{aligned}
\delta_{\zeta^{n}} h_{i j} & =\partial_{i} \zeta_{j}+\partial_{j} \zeta_{i}, \\
\delta_{\zeta} \pi^{i j} & =\partial^{i} \partial^{j} \zeta-\delta^{i j} \triangle \zeta .
\end{aligned}
$$

The Rarita-Schwinger action $S_{\mathrm{RS}}$ is given in (3.48).

The piece of the total action (5.1) containing only the fields $h_{m n}, \pi^{m n}, \mu_{m}$ and $\chi$ is invariant, up to a non-vanishing boundary terms, under rigid supersymmetry transformations of the form

$$
\begin{aligned}
\delta_{\epsilon_{0}} h_{m n}= & \frac{i}{2} \epsilon_{0}^{T} \gamma_{0} \gamma_{(m} \partial_{n)} \chi+\frac{i}{2} \epsilon_{0}^{T} \gamma_{0} \gamma_{(m} \mu_{n)}, \\
\delta_{\epsilon_{0}} \pi^{m n}= & \frac{i}{4} \epsilon_{0}^{T}\left(\partial^{m} \partial^{n} \chi-\delta^{m n} \Delta \chi\right) \\
& +\frac{i}{4} \epsilon_{0}^{T} \partial^{(m} \mu^{n)}-\frac{i}{4} \delta^{m n} \epsilon_{0}^{T} \partial_{k} \mu^{k}+\frac{i}{4} \epsilon_{0}^{T} \gamma_{0} \gamma_{5} \gamma^{(m} \epsilon^{n) p q} \partial_{p} \mu_{q}, \\
\delta_{\epsilon_{0}} \mu_{p}= & \frac{1}{4} \partial_{m} h_{n p} \gamma^{m n} \epsilon_{0}+\frac{1}{2} K_{m p} \gamma_{0} \gamma^{m} \epsilon_{0},
\end{aligned}
$$

and $\delta_{\epsilon_{0}} \chi=0$, where $\epsilon_{0}$ is a constant spinor parameter. In particular, the kinetic term is invariant but only up to a non-vanishing surface term.

The solution to the problem of achieving strict invariance is reached again by suitably choosing the transformation law under rigid supersymmetry for the boundary field $\rho^{k}$ in $S_{\mathrm{RS}}$, and by performing appropriate field dependent spin-2 gauge transformations with parameters

$$
\zeta_{\left(\epsilon_{0}\right)} \equiv i \epsilon_{0}^{T} \mathcal{F}, \quad \zeta_{\left(\epsilon_{0}\right)}^{n} \equiv i \epsilon_{0}^{T} \mathcal{F}^{n}, \quad\left(\Rightarrow \zeta_{\left(\epsilon_{0}\right)}=\zeta_{\left(\epsilon_{0}\right)}^{n}=0 \text { if } \epsilon_{0}=0\right),
$$

where $\mathcal{F}$ and $\mathcal{F}^{n}$ are functions of the fermionic dynamical fields, whose specific form is given in the next subsection. 


\subsection{Invariance under rigid supersymmetry}

The symplectic form for the multiplet $\left(2, \frac{3}{2}\right)$ reads as follows

$$
\Omega=\Omega_{\mathrm{PF}}+\Omega_{\mathrm{RS}},
$$

where

$$
\Omega_{\mathrm{PF}}=\int d^{3} x d_{V} \pi^{m n} d_{V} h_{m n}
$$

and $\Omega_{\mathrm{RS}}$ is given in (4.11).

The change in the symplectic form under rigid supersymmetry is given by

$$
\begin{aligned}
d_{V}\left(\iota_{\epsilon_{0}} \Omega\right)= & -\frac{i}{2} \oint d^{2} S_{m} \partial_{n} d_{V} \chi^{T} \epsilon_{0}\left(d_{V} h^{m n}-\delta^{m n} d_{V} h\right)-\frac{i}{2} \oint d^{2} S_{m} d_{V} \pi^{m n} d_{V} \chi^{T} \gamma_{0} \gamma_{n} \epsilon_{0} \\
& +\frac{i}{2} \alpha \oint d^{2} S_{m} d_{V} \chi^{T} \gamma_{0}\left(-\frac{1}{4} \gamma_{0} \gamma^{m n} \gamma^{p q} \partial_{p} d_{V} h_{n q} \epsilon_{0}+\frac{1}{2} d_{V} \pi^{m n} \gamma_{n} \epsilon_{0}+d_{V} \delta_{\epsilon_{0}} \rho^{m}\right) .
\end{aligned}
$$

The term proportional to $\alpha$ can be made zero, by imposing that the boundary field $\rho^{m}$ transforms as

$$
\delta_{\epsilon_{0}} \rho^{m}=\frac{1}{4} \gamma_{0} \gamma^{m n} \gamma^{p q} \partial_{p} h_{n q} \epsilon_{0}-\frac{1}{2} \pi^{m n} \gamma_{n} \epsilon_{0} \quad \Leftrightarrow \quad \delta_{\epsilon_{0}} \omega^{m}=0 .
$$

The remaining terms in (5.14) can be removed through the following field dependent spin-2 gauge transformations

$$
\begin{aligned}
d_{V}\left(\iota_{\zeta, \zeta^{k}} \Omega\right) & =\int d^{3} x\left(d_{V} \delta_{\zeta} \pi^{m n} d_{V} h_{m n}+d_{V} \pi^{m n} d_{V} \delta_{\zeta^{k}} h_{m n}\right) \\
& =2 \oint d^{2} S_{m} \partial_{n} d_{V} \zeta\left(d_{V} h^{m n}-\delta^{m n} d_{V} h\right)+2 \oint d^{2} S_{m} d_{V} \pi^{m n} d_{V} \zeta_{n}
\end{aligned}
$$

with parameters

$$
\zeta_{\left(\epsilon_{0}\right)}=\frac{i}{4} \chi^{T} \epsilon_{0}, \quad \zeta_{\left(\epsilon_{0}\right)}^{n}=\frac{i}{4} \chi^{T} \gamma_{0} \gamma^{n} \epsilon_{0} .
$$

We then obtain that the symplectic form is invariant

$$
d_{V}\left(\iota_{\epsilon_{0}} \Omega+\iota_{\zeta, \zeta^{n}} \Omega\right)=0
$$

which allows to define a canonical generator for rigid supersymmetry.

\subsection{Canonical realization of the asymptotic symmetries}

\subsubsection{Super-BMS algebra of $[14,15]$}

The Poincaré canonical generators are again just the sum of the individual Poincaré generators and read

$$
P_{\xi, \xi^{i}}=P_{\xi, \xi^{i}}^{\mathrm{PF}}+P_{\xi, \xi^{i}}^{\mathrm{RS}},
$$


where [38]

$$
\begin{aligned}
P_{\xi, \xi^{i}}^{\mathrm{PF}}= & \int d^{3} x\left(\xi \mathcal{H}^{\mathrm{PF}}+\xi^{i} \mathcal{H}_{i}^{\mathrm{PF}}\right)+\mathcal{B}_{\xi, \xi^{i}}^{\mathrm{PF}} \\
\mathcal{H}^{\mathrm{PF}}= & \pi^{i j} \pi_{i j}-\frac{\pi^{2}}{2}+\frac{1}{4} \partial_{k} h_{i j} \partial^{k} h^{i j}-\frac{1}{2} \partial_{j} h^{i j} \partial^{k} h_{i k}+\frac{1}{4} \partial_{i} h \partial^{i} h \\
& +\partial_{l}\left(-h^{i j} \partial^{l} h_{i j}-h^{i l} \partial_{i} h+\frac{3}{2} h^{l j} \partial^{i} h_{i j}+\frac{1}{2} h_{i j} \partial^{i} h^{j l}\right)-\frac{1}{2} h\left(\partial_{i} \partial_{j} h^{i j}-\triangle h\right) \\
\mathcal{H}_{i}^{\mathrm{PF}}= & -2 \partial_{j}\left(\pi^{j k} h_{i k}\right)+\pi^{j k} \partial_{i} h_{j k}, \\
\mathcal{B}_{\xi, \xi^{i}}^{\mathrm{PF}}= & \oint d^{2} x\left\{b\left[\sqrt{\bar{\gamma}}\left(-\frac{1}{2} \bar{h} \bar{h}_{r r}+\frac{1}{4} \bar{h}^{2}-\frac{3}{4} \bar{h}_{A B} \bar{h}^{A B}\right)+\frac{2}{\sqrt{\bar{\gamma}}} \bar{\pi}_{A}^{r} \bar{\pi}^{r A}\right]+2 Y_{A} \bar{\pi}^{r B} \bar{h}_{B}^{A}\right\},
\end{aligned}
$$

and $P_{\xi, \xi^{i}}^{\mathrm{RS}}$ is written in (3.63). Infinitesimal Poincaré transformations for Pauli-Fierz fields are given by

$$
\begin{aligned}
\delta_{\xi} h_{i j}= & 2 \xi\left(\pi_{i j}-\frac{1}{2} \delta_{i j} \pi\right)+\mathcal{L}_{\xi} h_{i j}, \\
\delta_{\xi} \pi^{i j}= & \frac{1}{2} \xi\left(\triangle h^{i j}+\partial^{i} \partial^{j} h-2 \partial_{k} \partial^{(i} h^{j) k}\right) \\
& \frac{1}{2} \partial_{k} \xi\left[\partial^{k} h^{i j}-2 \partial^{(i} h^{j) k}+\delta^{i j}\left(2 \partial_{l} h^{k l}-\partial^{k} h\right)\right] \\
& -\frac{1}{2} \delta^{i j} \xi\left(\triangle h-\partial_{i} \partial_{j} h^{i j}\right)+\mathcal{L}_{\xi} \pi^{i j},
\end{aligned}
$$

while the ones for Rarita-Schwinger fields are given in (3.60), (3.61) and (3.62).

The rigid supersymmetry canonical generator $G_{\epsilon_{0}}^{\mathrm{SUSY}}$, determined through $\iota_{\epsilon_{0}} \Omega+$ $\iota_{\zeta, \zeta^{n}} \Omega=-d_{V} G_{\epsilon_{0}}^{\mathrm{SUSY}}$ turns out to be given by

$$
G_{\epsilon_{0}}^{\mathrm{SUSY}}=i \int d^{3} x\left[-\frac{1}{2} \mu_{m}^{T} \pi^{m n} \gamma_{0} \gamma_{n} \epsilon_{0}-\frac{1}{4} \mu_{m}^{T}\left(\partial_{n} h^{m n}-\partial^{m} h\right) \epsilon_{0}-\frac{1}{4} \epsilon_{0}^{T} \gamma^{i} \gamma^{j r s} \mu_{s} \partial_{r} h_{i j}\right] .
$$

It reproduces through the Poisson bracket the above supersymmetry transformation laws for the fields accompanied by the above field-dependent spin-2 gauge transformations, i.e., explicitly,

$$
\begin{aligned}
\delta_{\epsilon_{0}} h_{m n} & =\frac{i}{2} \epsilon_{0}^{T} \gamma_{0} \gamma_{(m} \mu_{n)}, \\
\delta_{\epsilon_{0}} \pi^{m n} & =\frac{i}{4} \epsilon_{0}^{T} \partial^{(m} \mu^{n)}-\frac{i}{4} \delta^{m n} \epsilon_{0}^{T} \partial_{k} \mu^{k}+\frac{i}{4} \epsilon_{0}^{T} \gamma_{0} \gamma_{5} \gamma^{(m} \epsilon^{n) p q} \partial_{p} \mu_{q}, \\
\delta_{\epsilon_{0}} \mu_{p} & =-\frac{1}{4} \partial_{m} h_{n p} \gamma^{m n} \epsilon_{0}-\frac{1}{2} K_{m p} \gamma_{0} \gamma^{m} \epsilon_{0}, \\
\delta_{\epsilon_{0}} \rho^{m} & =\frac{1}{4} \gamma_{0} \gamma^{m n} \gamma^{p q} \partial_{p} h_{n q} \epsilon_{0}-\frac{1}{2} \pi^{m n} \gamma_{n} \epsilon_{0},
\end{aligned}
$$

and $\delta_{\epsilon_{0}} \chi=0$.

The canonical generator of the bosonic improper gauge transformations, which are the proper BMS supertranslations in the free theory [38], reads

$$
G_{T, W}=\int d^{3} x\left[\zeta\left(\triangle h-\partial_{i} \partial_{j} h^{i j}\right)-2 \zeta^{i} \partial_{j} \pi_{i}^{j}\right]+2 \oint d^{2} x\left[\sqrt{\bar{\gamma}} T \bar{h}_{r r}+W\left(\bar{\pi}^{r r}-\bar{\pi}_{A}^{A}\right)\right] .
$$


Infinitesimal gauge transformations are then given by

$$
\begin{aligned}
& \delta_{\zeta} h_{i j}=\partial_{i} \zeta_{j}+\partial_{j} \zeta_{i}, \\
& \delta_{\zeta} \pi^{i j}=\partial^{i} \partial^{j} \zeta-\delta^{i j} \triangle \zeta,
\end{aligned}
$$

with

$$
\zeta=T+\mathcal{O}\left(\frac{1}{r}\right), \quad \zeta^{i}=\partial^{i}(r W)+\mathcal{O}\left(\frac{1}{r}\right)
$$

where the set of functions $\left\{T^{\text {even }}, W^{\text {odd }}\right\}$ turns out to generate BMS supertranslations. The remaining parts $\left\{T^{\text {odd }}, W^{\text {even }}\right\}$ drop out trivially of the charge due to the parity conditions on the bosonic fields [32, 33], so these generate proper gauge transformations.

One may then easily derive the Poisson bracket algebra of the generators of the superPoincaré and BMS supertranslation generators, to get

$$
\begin{aligned}
\left\{P_{\xi_{1}, \xi_{1}^{i}}, P_{\xi_{2}, \xi_{2}^{i}}\right\} & =P_{\hat{\xi}, \hat{\xi}^{i}}, \\
\left\{P_{\xi, \xi^{i}}, G_{T, W}\right\} & =G_{\hat{T}, \hat{W}}, \\
\left\{G_{T_{1}, W_{1}}, G_{T_{2}, W_{2}}\right\} & =0, \\
\left\{P_{\xi, \xi^{i}}, G_{\epsilon_{0}}^{\mathrm{SUSY}}\right\} & =G_{\hat{\epsilon}_{0}}^{\mathrm{SUSY}}, \\
\left\{G_{\epsilon_{0}^{1}}^{\mathrm{SUY}}, G_{\epsilon_{0}^{2}}^{\mathrm{SUSY}}\right\} & =P_{\hat{a}, \hat{a}^{i}},
\end{aligned}
$$

where the parameters transform as

$$
\begin{array}{rlr}
\hat{T} & =-3 b W-\partial_{A} b \bar{D}^{A} W-b \bar{D}_{A} \bar{D}^{A} W+Y^{A} \partial_{A} T, \\
\hat{W} & =-b T+Y^{A} \partial_{A} W, \\
\hat{\epsilon}_{0} & =\frac{1}{2} \partial_{m} \xi \gamma^{m} \gamma_{0} \epsilon_{0}+\mathcal{L}_{\xi} \epsilon_{0}, \\
\hat{a} & =-\frac{i}{4}\left(\epsilon_{0}^{2}\right)^{T} \epsilon_{0}^{1}, \quad \hat{a}^{i}=-\frac{i}{4}\left(\epsilon_{0}^{2}\right)^{T} \gamma_{0} \gamma^{i} \epsilon_{0}^{1},
\end{array}
$$

with $\hat{\xi}$ and $\hat{\xi}^{i}$ given in (3.78) and (3.79), respectively.

This is just the super-BMS algebra of $[14,15]$, with the finite number of fermionic generators $G_{\epsilon_{0}}^{\text {SUSY }}$ parametrized by a constant spinor ("restricted super-BMS algebra"). To make the identification of the algebras, one must recall how the Poincare translations and the improper spin-2 gauge symmetries of the linear theory combine to form the full set of BMS super-translations [38].

\subsubsection{Infinite-dimensional fermionic gauge algebra}

But, as we have shown above, there are more fermionic symmetries, which take the form of improper gauge transformations. These asymptotic symmetries are clearly unaffected by the inclusion of the free spin-2- field. The full algebra, containing also these fermionic symmetries, is a graded extension of the BMS algebra with an infinite number of fermionic generators. 
The canonical generator of the improper fermionic gauge symmetries was worked out above,

$$
\begin{aligned}
G_{\epsilon, \varepsilon, \sigma^{k}}^{\mathrm{RS}}= & i \int d^{3} x\left(\epsilon^{T}+\varepsilon^{T}\right) \gamma^{k m} \partial_{k} \mu_{m} \\
& +\frac{i}{2} \alpha \oint d^{2} S_{k}\left(\epsilon^{T} \gamma^{k m} \mu_{m}-\chi^{T} \gamma^{k m} \partial_{m} \varepsilon-\epsilon^{T} \gamma_{0} \rho^{k}+\chi^{T} \gamma_{0} \sigma^{k}\right) .
\end{aligned}
$$

The brackets of this generator with the restricted super-BMS generators read

$$
\begin{aligned}
\left\{P_{\xi, \xi^{i}}, G_{\epsilon, \varepsilon, \sigma^{k}}^{\mathrm{RS}}\right\} & =G_{\hat{\epsilon}, \hat{\varepsilon}, \hat{\sigma}^{k}}^{\mathrm{RS}}, \\
\left\{G_{T, W}, G_{\epsilon, \varepsilon, \sigma^{k}}^{\mathrm{RS}}\right\} & =0, \\
\left\{G_{\epsilon_{0}}^{\mathrm{SUSY}}, G_{\epsilon, \varepsilon, \sigma^{k}}^{\mathrm{RS}}\right\} & =0,
\end{aligned}
$$

where $\hat{\epsilon}, \hat{\varepsilon}$ and $\hat{\sigma}$ are given in (3.80), (3.81) and (3.82), respectively. The brackets between the improper fermionic gauge symmetries form a centrally extended Abelian gauge algebra, written in (3.77).

\section{Conclusions}

In this paper, we have consistently relaxed the boundary conditions at spatial infinity of the Rarita-Schwinger field in such a way that the resulting fermionic improper gauge symmetries form an infinite-dimensional algebra parametrized by two independent functions of the angles. Poincaré invariance is maintained in the sense that not only the boundary conditions are Poincaré invariant but also the action itself so that the Poincaré transformations have well-defined (integrable and finite) generators. To achieve this result, we introduced boundary degrees of freedom at infinity, which modify the symplectic structure by boundary terms. We have also shown that the analysis can be extended to cover the supersymmetric free $(1,3 / 2)$ and $(2,3 / 2)$ multiplets and that it is compatible with supersymmetry. In the $(2,3 / 2)$ case, one finds a symmetry superalgebra which is graded extension of the BMS algebra with an infinite number of fermionic generators.

It remains to be explored whether similar results still hold when interactions are switched on, i.e. in supergravity. As the Yang-Mills example shows, this is not automatic [39]. Work along these lines is in progress.

\section{Acknowledgments}

O. F. holds a "Marina Solvay" fellowship. This work was partially supported by the ERC Advanced Grant "High-Spin-Grav", by FNRS-Belgium (conventions FRFC PDRT.1025.14 and IISN 4.4503.15), as well as by funds from the Solvay Family.

\section{A Notations and conventions}

Spinor fields will be mostly considered in two different orthonormal frames. One is the "cartesian frame" formed by the vectors $\left\{\frac{\partial}{\partial x^{i}}\right\}$. The other is the "spherical frame" given by $\left\{e_{(a)} k \frac{\partial}{\partial x^{k}} \equiv \frac{\partial}{\partial r}, \frac{1}{r} \frac{\partial}{\partial \theta}, \frac{1}{r \sin \theta} \frac{\partial}{\partial \varphi}\right\}$ in spherical coordinates. 
When dealing with the cartesian frame, it is natural to use cartesian coordinates. The associated Christoffel symbols and spin connection clearly vanish.

We give the corresponding formulas in the spherical frame, and use spherical coordinates. Indices in parentheses refer to the local frames, while indices without parentheses are coordinate indices. The matrix $\left(e_{(a)}^{k}\right)$ is given in spherical coordinates by

$$
\left(e_{(a)}{ }^{k}\right)=\left(\begin{array}{ccc}
1 & 0 & 0 \\
0 & \frac{1}{r} & 0 \\
0 & 0 & \frac{1}{r \sin \theta}
\end{array}\right) .
$$

The non-vanishing Christoffel symbols in spherical coordinates are

$$
\begin{aligned}
\Gamma_{\theta r}^{\theta} & =\Gamma_{r \theta}^{\theta}=\frac{1}{r}, & \Gamma^{r}{ }_{\theta \theta} & =-r, \\
\Gamma_{\varphi r}^{\varphi} & =\Gamma_{r \varphi}^{\varphi}=\frac{1}{r}, & \Gamma_{\varphi \varphi}^{r} & =-r \sin ^{2} \theta, \\
\Gamma_{\varphi \theta}^{\varphi} & =\Gamma^{\varphi}{ }_{\theta \varphi}=\frac{\cos \theta}{\sin \theta}, & \Gamma^{\theta} \varphi & =-\cos \theta \sin \theta,
\end{aligned}
$$

while the spin connection coefficients $\omega_{(a)(b) m}=-\omega_{(b)(a) m}$ defined as

$$
\omega_{(a)(b) m}=e_{(a) k \mid m} e_{(b)}^{k},
$$

fulfill the triad condition

$$
\nabla_{m} e_{(a)}^{k}=e_{(a) \mid m}^{k}-\omega_{(a) m}^{(b)} e_{(b)}^{k}=0,
$$

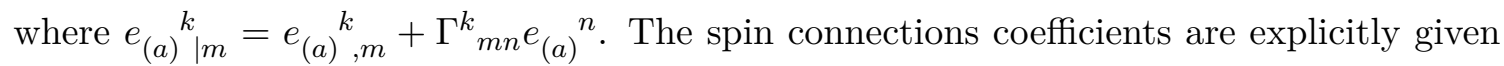
in the spherical frame by

$$
\begin{array}{lll}
\omega_{(a)(b) r}=0, & \\
\omega_{(1)(2) \theta}=1=-\omega_{(2)(1) \theta}, & \omega_{(1)(3) \theta}=0=-\omega_{(3)(1) \theta}, & \omega_{(2)(3) \theta}=0=-\omega_{(3)(2) \theta}, \\
\omega_{(1)(2) \varphi}=0=-\omega_{(2)(1) \varphi}, & \omega_{(1)(3) \varphi}=\sin \theta=-\omega_{(3)(1) \varphi}, & \omega_{(2)(3) \varphi}=\cos \theta=-\omega_{(3)(2) \varphi},
\end{array}
$$

The $\gamma$-matrices are

$$
\begin{aligned}
\gamma_{(0)}=\left(\begin{array}{cccc}
0 & 1 & 0 & 0 \\
-1 & 0 & 0 & 0 \\
0 & 0 & 0 & -1 \\
0 & 0 & 1 & 0
\end{array}\right), & \gamma_{(1)}=\left(\begin{array}{cccc}
1 & 0 & 0 & 0 \\
0 & -1 & 0 & 0 \\
0 & 0 & -1 & 0 \\
0 & 0 & 0 & 1
\end{array}\right) \\
\gamma_{(2)}=\left(\begin{array}{cccc}
0 & 0 & -1 & 0 \\
0 & 0 & 0 & -1 \\
-1 & 0 & 0 & 0 \\
0 & -1 & 0 & 0
\end{array}\right) & \gamma_{(3)}=\left(\begin{array}{cccc}
0 & -1 & 0 & 0 \\
-1 & 0 & 0 & 0 \\
0 & 0 & 0 & 1 \\
0 & 0 & 1 & 0
\end{array}\right),
\end{aligned}
$$

where $\gamma_{(r)}$ coincides in the spherical frame with $\gamma_{(1)}$. The spin covariant derivative of the $\gamma$-matrices in spherical coordinates $\gamma_{m}$, and in the local frame $\gamma_{(a)}$,

$$
\begin{aligned}
& \nabla_{k} \gamma_{m}=\partial_{k} \gamma_{k}-\Gamma_{k m}^{n} \gamma_{n}-\frac{1}{4} \omega_{(c)(d) k}\left[\gamma^{(c)(d)}, \gamma_{m}\right]=0, \\
& \nabla_{k} \gamma_{(a)}=\partial_{k} \gamma_{(a)}-\frac{1}{4} \omega_{(c)(d) k}\left[\gamma^{(c)(d)}, \gamma_{(a)}\right]-\omega_{(a)(b) k} \gamma^{(b)}=0,
\end{aligned}
$$


vanish. The covariant derivative of spinor fields and vector-spinor fields are respectively given by

$$
\begin{aligned}
\nabla_{k} \chi & =\partial_{k} \chi-\frac{1}{4} \omega_{(a)(b) k} \gamma^{(a)(b)} \chi \\
\nabla_{k} \mu_{m} & =\partial_{k} \mu_{m}-\Gamma_{k m}^{n} \mu_{n}-\frac{1}{4} \omega_{(a)(b) k} \gamma^{(a)(b)} \mu_{m}
\end{aligned}
$$

\section{B Asymptotic conditions and charges in spherical coordinates}

In spherical coordinates, the radial and angular components of the spinor fields fall-off as

$$
\begin{array}{rlrl}
\chi & =\bar{\chi}+\mathcal{O}\left(\frac{1}{r}\right), & \\
\mu_{r}=\frac{\bar{\mu}_{r}}{r^{2}}+\mathcal{O}\left(\frac{1}{r^{3}}\right), & \mu_{A}=\frac{\bar{\mu}_{A}}{r}+\mathcal{O}\left(\frac{1}{r^{2}}\right), \\
\rho_{r}=\frac{\bar{\rho}_{r}}{r^{2}}+\mathcal{O}\left(\frac{1}{r^{3}}\right), & \rho_{A}=\frac{\bar{\rho}_{A}}{r}+\mathcal{O}\left(\frac{1}{r^{2}}\right) .
\end{array}
$$

The Poincaré parameters behave as

$$
\xi=r b+T, \quad \xi^{r}=W, \quad \xi^{A}=Y^{A}+\frac{1}{r} \bar{D}^{A} W
$$

where the asymptotic Killing equations read

$$
\bar{D}^{A} \bar{D}^{B} W+\bar{\gamma}_{A B} W=0, \quad \bar{D}_{A} \bar{D}_{B} b+\bar{\gamma}_{A B} b=0, \quad \mathcal{L}_{Y} \bar{\gamma}_{A B}=0, \quad \partial_{A} T=0
$$

The functions on the sphere, $b$ and $Y^{A}$ describe the homogeneous Lorentz transformations, while $T$ and $W$ describe translations. Here, $\bar{D}^{A}$ denotes the covariant derivative with respect to the metric on the 2 -sphere. The gauge parameters behave as

$$
\begin{aligned}
\epsilon & =\bar{\epsilon}+\mathcal{O}\left(\frac{1}{r}\right), \\
\varepsilon & =\frac{\bar{\varepsilon}}{r}+\mathcal{O}\left(\frac{1}{r^{2}}\right), \\
\sigma_{r} & =\frac{\bar{\sigma}_{r}}{r^{2}}+\mathcal{O}\left(\frac{1}{r^{3}}\right), \\
\sigma_{A} & =\frac{\bar{\sigma}_{A}}{r}+\mathcal{O}\left(\frac{1}{r^{2}}\right) .
\end{aligned}
$$

The explicit form of the constraint in spherical coordinates at leading order reads

$$
\mathcal{S}=\frac{1}{r}\left(\bar{\gamma}^{A B} \bar{\nabla}_{A} \bar{\mu}_{B}-\frac{1}{2} \gamma_{1} \bar{\gamma}^{A} \bar{\mu}_{A}-\gamma^{r} \bar{\gamma}^{A} \bar{\nabla}_{A} \bar{\mu}_{r}-\bar{\mu}_{r}\right)+\mathcal{O}\left(\frac{1}{r^{2}}\right)
$$

where $\bar{\nabla}_{A}$ stands for the spin-covariant derivative on the 2 -sphere. 
Poincaré and fermionic gauge charges in spherical coordinates. Poincaré generators are given by

$$
P_{\xi, \xi^{i}}=\int d^{3} x\left(\xi \mathcal{H}^{\mathrm{RS}}+\xi^{i} \mathcal{H}_{i}^{\mathrm{RS}}\right)+\mathcal{B}_{\xi, \xi^{i}}^{\mathrm{RS}}
$$

where the bulk pieces of the generators can be read off from (3.63), and the asymptotic charges become

$$
\mathcal{B}_{\xi, \xi^{i}}^{\mathrm{RS}}=\frac{i}{2} \alpha \oint d^{2} x\left(\delta_{b} \bar{\chi}^{T} \gamma_{0} \bar{\omega}^{r}+\mathcal{L}_{Y} \bar{\chi}^{T} \gamma_{0} \bar{\omega}^{r}\right)
$$

Infinitesimal transformation laws under Poincaré are determined by

$$
\begin{aligned}
\delta \bar{\chi}= & -b \gamma_{0} \bar{\gamma}^{A} \bar{D}_{A} \bar{\chi}+\frac{3}{2} b \gamma^{r} \gamma_{0} \bar{\chi}+\frac{1}{2} \bar{\nabla}_{B} b \bar{\gamma}^{B} \gamma_{0} \bar{\chi}+\mathcal{L}_{Y} \bar{\chi}, \\
\delta \bar{\mu}_{r}= & b \gamma_{0} \gamma^{r}\left(\bar{\gamma}^{B C} \bar{\nabla}_{B} \bar{\mu}_{C}+\gamma^{r} \bar{\gamma}^{B} \bar{\mu}_{B}\right)+\frac{1}{2} b \gamma^{r} \gamma_{0} \bar{\mu}_{r}+\frac{1}{2} \bar{\nabla}_{B} b \bar{\gamma}^{B} \gamma_{0} \bar{\mu}_{r} \\
& +\frac{1}{2} b \gamma_{0} \gamma^{r}\left(\bar{\gamma}^{A B} \bar{\nabla}_{A} \bar{\mu}_{B}-\gamma^{r} \bar{\gamma}^{A} \bar{\nabla}_{A} \bar{\mu}_{r}-\bar{\mu}_{r}\right)+\mathcal{L}_{Y} \bar{\mu}_{r}, \\
\delta \bar{\mu}_{A}= & b \gamma_{0} \bar{\gamma}_{A}^{B C} \bar{\nabla}_{B} \bar{\mu}_{C}+b \gamma_{0} \gamma^{r} \bar{\gamma}_{A}^{B}\left(\bar{\nabla}_{B} \bar{\mu}_{r}-\frac{1}{2} \gamma^{r} \bar{\gamma}_{B} \bar{\mu}_{r}-\frac{1}{2} \bar{\mu}_{B}\right) \\
& +\frac{1}{2} b \gamma_{0} \bar{\gamma}_{A}\left(\bar{\gamma}^{B C} \bar{\nabla}_{B} \bar{\mu}_{C}-\gamma^{r} \bar{\gamma}^{B} \bar{\nabla}_{B} \bar{\mu}_{r}-\bar{\mu}_{r}\right)+\mathcal{L}_{Y} \bar{\mu}_{A}, \\
\delta \bar{\rho}^{r}= & -\gamma^{r} \bar{D}_{A}\left(b \bar{\mu}^{A}\right)-\frac{1}{2} b \bar{\gamma}_{A} \bar{\mu}^{A}+b \gamma_{0} \bar{\gamma}^{A} \bar{D}_{A} \bar{\rho}^{r}+\frac{3}{2} b \gamma^{r} \gamma_{0} \bar{\rho}^{r} \\
& -\frac{1}{2} \partial_{A} b \bar{\gamma}^{A} \gamma_{0} \bar{\rho}^{r}+\mathcal{L}_{Y} \bar{\rho}^{r}, \\
\delta \bar{\rho}^{A}= & 0 .
\end{aligned}
$$

The surface charges of improper gauge symmetries are

$$
\mathcal{B}_{\epsilon, \varepsilon, \sigma^{k}}^{\mathrm{RS}}=\frac{i}{2} \alpha \oint d^{2} x\left(\bar{\epsilon}^{T} \gamma^{r} \bar{\gamma}^{A} \bar{\mu}_{A}-\bar{\chi}^{T} \gamma^{r} \bar{\gamma}^{A} \bar{D}_{A} \bar{\varepsilon}-\bar{\epsilon}^{T} \gamma_{0} \bar{\rho}^{r}+\bar{\chi}^{T} \gamma_{0} \bar{\sigma}^{r}\right),
$$

where the infinitesimal transformation laws under gauge symmetries at leading order are given by

$$
\delta_{\epsilon} \bar{\chi}=\bar{\epsilon}, \quad \delta_{\varepsilon} \bar{\mu}_{r}=-\bar{\varepsilon}, \quad \delta_{\varepsilon} \bar{\mu}_{A}=\partial_{A} \bar{\varepsilon}, \quad \delta_{\sigma} \bar{\rho}_{r}=\bar{\sigma}_{r}, \quad \delta_{\sigma} \bar{\rho}_{A}=\bar{\sigma}_{A} .
$$

Open Access. This article is distributed under the terms of the Creative Commons Attribution License (CC-BY 4.0), which permits any use, distribution and reproduction in any medium, provided the original author(s) and source are credited.

\section{References}

[1] A. Strominger, Lectures on the infrared structure of gravity and gauge theory, arXiv: 1703.05448 [INSPIRE].

[2] H. Bondi, M.G.J. van der Burg and A.W.K. Metzner, Gravitational waves in general relativity. 7. Waves from axisymmetric isolated systems, Proc. Roy. Soc. Lond. A 269 (1962) 21 [INSPIRE]. 
[3] R.K. Sachs, Gravitational waves in general relativity. 8. Waves in asymptotically flat space-times, Proc. Roy. Soc. Lond. A 270 (1962) 103 [InSPIRE].

[4] R. Sachs, Asymptotic symmetries in gravitational theory, Phys. Rev. 128 (1962) 2851 [INSPIRE].

[5] R. Penrose, Asymptotic properties of fields and space-times, Phys. Rev. Lett. 10 (1963) 66 [INSPIRE].

[6] T. Mädler and J. Winicour, Bondi-Sachs formalism, Scholarpedia 11 (2016) 33528 [arXiv: 1609.01731] [INSPIRE].

[7] F. Alessio and G. Esposito, On the structure and applications of the Bondi-Metzner-Sachs group, Int. J. Geom. Meth. Mod. Phys. 15 (2018) 1830002 [arXiv:1709.05134] [InSPIRE].

[8] A. Ashtekar, M. Campiglia and A. Laddha, Null infinity, the BMS group and infrared issues, Gen. Rel. Grav. 50 (2018) 140 [arXiv:1808. 07093] [InSPIRE].

[9] T. Banks, A critique of pure string theory: heterodox opinions of diverse dimensions, hep-th/0306074 [INSPIRE].

[10] G. Barnich and C. Troessaert, Symmetries of asymptotically flat 4 dimensional spacetimes at null infinity revisited, Phys. Rev. Lett. 105 (2010) 111103 [arXiv:0909.2617] [INSPIRE].

[11] G. Barnich and C. Troessaert, Aspects of the BMS/CFT correspondence, JHEP 05 (2010) 062 [arXiv: 1001.1541] [INSPIRE].

[12] G. Barnich and C. Troessaert, Supertranslations call for superrotations, PoS CNCFG2010 (2010) 010 [Ann. U. Craiova Phys. 21 (2011) S11] [arXiv:1102.4632] [InSPIRE].

[13] J.D. Brown and M. Henneaux, Central charges in the canonical realization of asymptotic symmetries: an example from three-dimensional gravity, Commun. Math. Phys. 104 (1986) 207 [INSPIRE].

[14] M.A. Awada, G.W. Gibbons and W.T. Shaw, Conformal supergravity, twistors and the super BMS group, Annals Phys. 171 (1986) 52 [INSPIRE].

[15] M. Henneaux, J. Matulich and T. Neogi, Asymptotic realization of the super-BMS algebra at spatial infinity, Phys. Rev. D 101 (2020) 126016 [arXiv:2004.07299] [INSPIRE].

[16] M. Bañados, K. Bautier, O. Coussaert, M. Henneaux and M. Ortiz, Anti-de Sitter/CFT correspondence in three-dimensional supergravity, Phys. Rev. D 58 (1998) 085020 [hep-th/9805165] [INSPIRE].

[17] M. Henneaux, L. Maoz and A. Schwimmer, Asymptotic dynamics and asymptotic symmetries of three-dimensional extended AdS supergravity, Annals Phys. 282 (2000) 31 [hep-th/9910013] [INSPIRE].

[18] T.T. Dumitrescu, T. He, P. Mitra and A. Strominger, Infinite-dimensional fermionic symmetry in supersymmetric gauge theories, arXiv:1511.07429 [INSPIRE].

[19] V. Lysov, Asymptotic fermionic symmetry from soft gravitino theorem, arXiv:1512.03015 [INSPIRE].

[20] S.G. Avery and B.U.W. Schwab, Residual local supersymmetry and the soft gravitino, Phys. Rev. Lett. 116 (2016) 171601 [arXiv: 1512.02657] [INSPIRE].

[21] A. Fotopoulos, S. Stieberger, T.R. Taylor and B. Zhu, Extended super BMS algebra of celestial CFT, JHEP 09 (2020) 198 [arXiv:2007.03785] [INSPIRE]. 
[22] S.A. Narayanan, Massive celestial fermions, JHEP 12 (2020) 074 [arXiv: 2009.03883] [INSPIRE].

[23] G. Barnich, L. Donnay, J. Matulich and R. Troncoso, Asymptotic symmetries and dynamics of three-dimensional flat supergravity, JHEP 08 (2014) 071 [arXiv:1407.4275] [INSPIRE].

[24] N. Banerjee, D.P. Jatkar, I. Lodato, S. Mukhi and T. Neogi, Extended supersymmetric BMS algebras and their free field realisations, JHEP 11 (2016) 059 [arXiv: 1609. 09210] [INSPIRE].

[25] I. Lodato and W. Merbis, Super-BMS $S_{3}$ algebras from $\mathcal{N}=2$ flat supergravities, JHEP 11 (2016) 150 [arXiv: 1610.07506] [inSPIRE].

[26] N. Banerjee, I. Lodato and T. Neogi, $N=4$ Supersymmetric $B M S_{3}$ algebras from asymptotic symmetry analysis, Phys. Rev. D 96 (2017) 066029 [arXiv:1706.02922] [INSPIRE].

[27] R. Basu, S. Detournay and M. Riegler, Spectral flow in 3D flat spacetimes, JHEP 12 (2017) 134 [arXiv: 1706.07438] [INSPIRE].

[28] O. Fuentealba, J. Matulich and R. Troncoso, Asymptotic structure of $\mathcal{N}=2$ supergravity in 3D: extended super-BMS $S_{3}$ and nonlinear energy bounds, JHEP 09 (2017) 030 [arXiv: 1706.07542] [INSPIRE].

[29] R. Caroca, P. Concha, O. Fierro and E. Rodríguez, Three-dimensional Poincaré supergravity and $N$-extended supersymmetric $B M S_{3}$ algebra, Phys. Lett. B 792 (2019) 93 [arXiv: 1812.05065] [INSPIRE].

[30] R. Caroca, P. Concha, O. Fierro and E. Rodríguez, On the supersymmetric extension of asymptotic symmetries in three spacetime dimensions, Eur. Phys. J. C 80 (2020) 29 [arXiv: 1908.09150] [INSPIRE].

[31] M. Henneaux and C. Troessaert, BMS group at spatial infinity: the hamiltonian (ADM) approach, JHEP 03 (2018) 147 [arXiv:1801.03718] [INSPIRE].

[32] M. Henneaux and C. Troessaert, Hamiltonian structure and asymptotic symmetries of the Einstein-Maxwell system at spatial infinity, JHEP 07 (2018) 171 [arXiv:1805.11288] [INSPIRE].

[33] M. Henneaux and C. Troessaert, The asymptotic structure of gravity at spatial infinity in four spacetime dimensions, arXiv:1904.04495 [INSPIRE].

[34] H. Friedrich, Smoothness at null infinity and the structure of initial data, in The Einstein equations and large scale behaviour of gravitational fields, P.T. Chruściel and H. Friedrich eds., Birkhäuser, Basel Swizerland (2004).

[35] J.A. Valiente-Kroon, A new class of obstructions to the smoothness of null infinity, Commun. Math. Phys. 244 (2004) 133 [gr-qc/0211024] [InSPIRE].

[36] M. Henneaux and C. Troessaert, Asymptotic symmetries of electromagnetism at spatial infinity, JHEP 05 (2018) 137 [arXiv:1803.10194] [INSPIRE].

[37] T. Regge and C. Teitelboim, Role of surface integrals in the Hamiltonian formulation of general relativity, Annals Phys. 88 (1974) 286 [INSPIRE].

[38] O. Fuentealba, M. Henneaux, S. Majumdar, J. Matulich and C. Troessaert, Asymptotic structure of the Pauli-Fierz theory in four spacetime dimensions, Class. Quant. Grav. 37 (2020) 235011 [arXiv:2007.12721] [INSPIRE].

[39] R. Tanzi and D. Giulini, Asymptotic symmetries of Yang-Mills fields in Hamiltonian formulation, JHEP 10 (2020) 094 [arXiv: 2006. 07268] [INSPIRE]. 
[40] R. Benguria, P. Cordero and C. Teitelboim, Aspects of the Hamiltonian dynamics of interacting gravitational gauge and Higgs fields with applications to spherical symmetry, Nucl. Phys. B 122 (1977) 61 [inSPIRE].

[41] J.-L. Gervais, B. Sakita and S. Wadia, The surface term in gauge theories, Phys. Lett. B 63 (1976) 55 [INSPIRE].

[42] L. Freidel, M. Geiller and D. Pranzetti, Edge modes of gravity. Part I. Corner potentials and charges, JHEP 11 (2020) 026 [arXiv:2006.12527] [INSPIRE].

[43] L. Freidel, M. Geiller and D. Pranzetti, Edge modes of gravity. Part II. Corner metric and Lorentz charges, JHEP 11 (2020) 027 [arXiv: 2007.03563] [INSPIRE].

[44] L. Freidel, M. Geiller and D. Pranzetti, Edge modes of gravity. Part III. Corner simplicity constraints, JHEP 21 (2020) 100 [arXiv:2007.12635] [INSPIRE].

[45] S. Deser, J.H. Kay and K.S. Stelle, Hamiltonian formulation of supergravity, Phys. Rev. D 16 (1977) 2448 [INSPIRE].

[46] M. Henneaux and C. Troessaert, Asymptotic structure of electromagnetism in higher spacetime dimensions, Phys. Rev. D 99 (2019) 125006 [arXiv: 1903.04437] [InSPIRE].

[47] M. Henneaux and C. Troessaert, A note on electric-magnetic duality and soft charges, JHEP 06 (2020) 081 [arXiv: 2004.05668] [INSPIRE].

[48] J.D. Brown and M. Henneaux, On the Poisson brackets of differentiable generators in classical field theory, J. Math. Phys. 27 (1986) 489 [InSPIRE]. 